Review

\title{
Overview of stimuli-responsive mesoporous organosilica nanocarriers for drug delivery
}

\author{
Rafaela S. Guimarães $^{\mathrm{a}}$, Carolina F. Rodrigues ${ }^{\mathrm{a}}$, André F. Moreira ${ }^{\mathrm{a}, * *}$, Ilídio J. Correia ${ }^{\mathrm{a}, \mathrm{b}, *}$ \\ ${ }^{a}$ CICS-UBI - Health Sciences Research Centre, Universidade da Beira Interior, Av. Infante D. Henrique, 6200-506, Covilhã, Portugal \\ ${ }^{\text {b } C I E P Q F}$ - Departamento de Engenharia Química, Universidade de Coimbra, Rua Sílvio Lima, 3030-790, Coimbra, Portugal
}

\section{A R T I C L E I N F O}

\section{Keywords:}

Mesoporous organosilica nanoparticles

Drug delivery

Cancer therapy

Nanomedicine

Stimuli responsive

\begin{abstract}
A B S T R A C T
The application of nanomaterials is regarded nowadays as a highly promising approach for overcoming the limitations of the currently available cancer treatments, contributing for the creation of more effective, precise, and safer therapies. In the last years, organosilica nanoparticles arisen as alternatives to the most common mesoporous silica nanoparticles. The organosilica nanoparticles combine the advantages of the mesoporous silica, such as structural stability and mesoporous structure, with the increased biocompatibility and biodegradability of organic materials. Therefore, the variety of organic bridges that can be incorporated into the silica matrix allowed the development of new and exciting compositions, properties, and functions for improving the therapeutic effectiveness of the anticancer nanomedicines.

In this review, the strategies that have been explored to create stimuli-responsive organosilica-based drug delivery systems are highlighted, describing the practical approaches and mechanisms controlling the drug release. Additionally, the organosilica nanoparticles surface modifications aimed for increasing the blood circulation time and the tumor targeting are also described.
\end{abstract}

\section{Introduction}

In recent years, nanomaterials have been widely employed to develop new biomedical solutions such as biosensors, 3D structures for tissue engineering, and targeted drug delivery systems [1,2]. Particularly, the development of nanoparticles for cancer therapeutic applications has been one of the most active fields in nanomedicine. These approaches take advantage of the nanoparticles innate capacity to accumulate on the tumor tissue and, therefore, overcome the main limitations associated with cancer conventional therapies (e.g. non-specific toxicity, low bioavailability, and lack of efficacy) [2,3]. Such feature prompted the development of a plethora of nanomaterials that can be composed of organic (e.g. lipids, polymers, and proteins) and inorganic (e.g. carbon, gold, and silica) materials [4-8].

Among them, the mesoporous silica nanoparticles (MSNs) have attracted the attention of the scientific community due to its simple and easily scalable synthesis that allows the production of particles with large surface area, well-defined ordered structure, and tunable pore size [9]. Specially, the MSNs' characteristic pore structure allows the encapsulation of large amounts of therapeutic agents, protecting them from premature degradation and avoiding their interaction with healthy tissues [10-14]. On the other hand, the introduction of pore gatekeepers (e.g. polymers, nanoparticles or small molecules) creates a drug delivery system that is capable of controlling, both in space and time, the drug release in order to increase the antitumoral effect (reviewed in detail by $[2,15,16])$. Nevertheless, there are still crucial parameters, such as the MSNs' biodegradation, biosafety, and excretion, that need to be precisely tuned to allow its broad application in the nanomedicine field $[17,18]$.

In this way, considering the high biocompatibility and biodegradability observed in organic drug delivery systems (e.g. liposomes, solidlipid nanoparticles, and micelles), researchers started to explore the introduction of organic moieties into the inorganic silica (Si-O-Si) matrix $[19,20]$. Further, the almost unlimited options of organosilane precursors opens the possibility to design and optimize the nanoparticles physicochemical properties and biological performance $[21,22]$. Such impelled the creation of a wide number of hybrid silica structures that can be grouped in two main categories, the periodic mesoporous organosilicas (PMOs - produced using only organosilica precursors) and mesoporous organosilica nanoparticles (MONs - combine both conventional silica and organosilica precursors) [23,24]. In general, the organosilica-based nanoparticles can maintain some

\footnotetext{
* Corresponding author at: CICS-UBI - Health Sciences Research Centre, Universidade da Beira Interior, Av. Infante D. Henrique, 6200-506, Covilhã, Portugal.

*** Corresponding author.

E-mail addresses: afmoreira@fcsaude.ubi.pt (A.F. Moreira), icorreia@ubi.pt (I.J. Correia).
} 
properties of the original MSNs, such as the hydrothermal and hydrolytic stability as well as the ordered porous structure, and combine them with an improved colloidal stability, biodegradability, biocompatibility and stimuli-responsiveness $[25,26]$. In fact, this organosilica structural plasticity allowed the development of various nanomedicine-based solutions for bioimaging (e.g. magnetic resonance, fluorescent, and ultrasounds) and therapeutic (e.g. controlled drug delivery, immunotherapy, and tissue engineering) applications $[20,25,27-29]$. In this article, the most recent progresses in organosilica-based nanoparticles for drug delivery in cancer therapy are reviewed. Moreover, the different strategies employed for conferring them a stimuli-responsive drug release profile and a tumor-specific bioaccumulation will be highlighted. Finally, an overview of the organosilica-based nanoparticles' biosafety and biocompatibility is also provided.

\section{Organosilica nanoparticles}

The organosilica nanoparticles physico-chemical properties, structural plasticity, and biocompatibility makes them one of the most promising hybrid materials for therapeutic applications. In fact, the organosilica nanomaterials present a controllable size, porous structure, and pore size [30]. Further, the wide range of organosilica precursors also allow the researchers to fine-tune the nanoparticle composition, surface chemistry and dispersibility [22,31]. Additionally, the large pore volume and tunable pore size allows the encapsulation of increased payloads of therapeutic agents such as chemotherapeutic drugs or even larger molecules like genetic material and proteins [21,32-35]. Such, combined with a stimuli-sensitive particle degradation, mediated by the organic constituents present on the particles' matrix, can be explored for increasing the treatments specificity and avoid the rapid in vivo degradation of the therapeutic agents $[32,36]$. Similarly, the possibility to control the particle degradation rate also increase the control over the organosilica nanoparticles half-life in the human body and clearance rates, improving the biosafety of these drug delivery systems [37]. On the other hand, the structural plasticity of the organosilica nanoparticles can also be explored to imprint new functionalities in the nanomedicines such as fluorescence, photodynamic capacity, or even tumor targeting [38-43]. Another advantage of organosilica nanoparticles is the commercial availability of several precursors and the scalability of the synthesis procedure, which allows the large-scale production and facilitates the translation of these nanomedicines into the market.

\subsection{Synthesis of organosilica nanoparticles}

The organosilica nanoparticles (PMOs and MONs) like the MSNs are usually produced using sol-gel processes (Fig. 1) [24,44]. In general, during this process occurs the hydrolysis and subsequent condensation of silane molecules in an aqueous solution under basic or acidic conditions and in the presence of a soft pore template (i.e. surfactants or amphiphilic polymers) [30]. The base catalysed hydrolysis of the silane precursors, tetraalkyl silicates $\left(\mathrm{Si}\left(\mathrm{OX}_{4}\right)\right.$, where $\mathrm{X}$ is typically OEt or $\mathrm{OMe}$ ) or organosilanes $\left(\left[(\mathrm{XO})_{3} \mathrm{Si}\right]_{\mathrm{n}}-\mathrm{R}\right.$, where $\mathrm{R}$ is an organic group, $\mathrm{n} \geq$ 1 ), creates reactive silanolate species that will condensate and form stable covalent siloxane bonds with other silane molecules [45]. It is worth to notice that the organic group can vary from simple organic bridges (e.g. methylene, ethylene, and propylene) to complex ones (e.g. aromatic and long hydrocarbon chains composed of $\mathrm{N}, \mathrm{S}$ or $\mathrm{O}$ ) $[1,17,20]$. The condensation of several tetraalkyl silicates originates silica structures $\left(\mathrm{SiO}_{2}\right)$, whereas in the presence of organosilane precursors originate silsesquioxane $\left(\left[\mathrm{RSiO}_{3 / 2}\right]_{\mathrm{n}}\right)$ matrices [46]. Additionally, the soft pore template (e.g. hexadecyltrimethylammonium bromide (CTAB)) direct the silane precursors condensation to their surface, usually by exploring the electrostatic interaction between the positively charged pore template and the negatively charged silanolate species [2]. In this way, the packing of these coated pore template structures results in the formation of the nanoparticles [9]. If a mixture of tetraalkyl silicates and organosilanes is used the particles are usually denominated as MONs, whereas the solo utilization of organosilanes originates the PMOs [47-49]. After the organosilica nanoparticles' synthesis, the soft pore template must be removed to allow the subsequent loading of the therapeutic agents and due to biocompatibility issues (e.g. the CTAB is highly cytotoxic) $[47,49]$. In contrast to the MSNs, the purification of organosilica nanoparticles demands the utilization of less aggressive extraction procedures in order to preserve the organic content [30]. Therefore, the solvent extraction or dialysis approaches have been the most explored extraction protocols for both MONs and PMOs [50-52].

Nevertheless, it is important to refer that the introduction of the organosilanes on the sol-gel process is still a challenging step. In fact, the size, electric charge, chemical composition and wettability of the organosilane precursors are crucial factors that influence the interaction of the silanolate species with the pore template agents. Such interaction is of paramount importance for the characteristic porous structure of the nanoparticles or even for the synthesis of uniform and monodisperse organosilica nanoparticles. In this way, the selection of the organosilane precursor is crucial for the production of nanoparticles aimed for biomedical applications. For example, Guan and colleagues observed that the alteration on the organosilane precursor from methylene to ethylene, ethenylene, or phenylene resulted in the formation of PMOs with different porous structures, 3D hexagonal (P63/mmc), cubic (Pm3n), 2D hexagonal (P6mm), and wormlike pores, respectively [53]. Similarly, Croissant et al. described the formation of PMOs with different morphologies by using 1,4-bis(triethoxysilyl)ethylene, bis (3-ethoxysilylpropyl)disulphide, or its combination [54]. The authors reported that the single utilization of 1,4- bis(triethoxysilyl)ethylene resulted in the formation of rod-shaped porous PMOs, whereas the bis (3-ethoxysilylpropyl)disulphide produced non-porous spherical PMOs. Moreover, the authors also described the production rod-shaped porous PMOs with different sizes by combining the two different organosilane precursors. In the Table 1, it is provided an overview of the different organosilica precursors used, synthesis approach, and the final properties of the nanoparticles such as the particle size and charge.

\section{Organosilica nanoparticles application in cancer therapy}

The organosilica nanoparticles porous structure conjugated with their structural plasticity can be explored to introduce new functionalities such as fluorescence, photodynamic capacity, or even tumor targeting that support the application of these nanomedicines in cancer therapy [45,63]. Particularly, in tumor drug delivery applications, the presence of the organic bridges on the silica matrix impacts on their chemical properties, which can be explored to tune the hydrophobicity/ hydrophilicity or even the surface charge of the pore walls in order to maximize the drug loading $[20,65,66]$. In this way, the drug encapsulation in the organosilica nanoparticles pores can be achieved through nanoparticles/drug dispersion methods in order to stimulate electrostatic or hydrophobic/hydrophilic interactions between the therapeutic molecules and the nanoparticle pores [47,67].

On the other hand, the same organic bridges can also mediate the therapeutics release from the organosilica nanoparticles through stimuli dependent mechanisms, such as $\mathrm{pH}$, redox potential, and temperature, that trigger the nanoparticle disassembly/destruction (Fig. 2) [20,67]. Additionally, the organosilica nanoparticles' surface can be modified with stimuli-responsive moieties that will work as pore blocking agents and mediate the drug release in the presence of a specific stimulus [68]. This superior control over the drug release is paramount to reduce the interaction with healthy tissues and enhance the therapeutic outcome $[69,70]$.

In this section, the different approaches explored in the literature to develop stimuli-responsive organosilica drug delivery systems is 


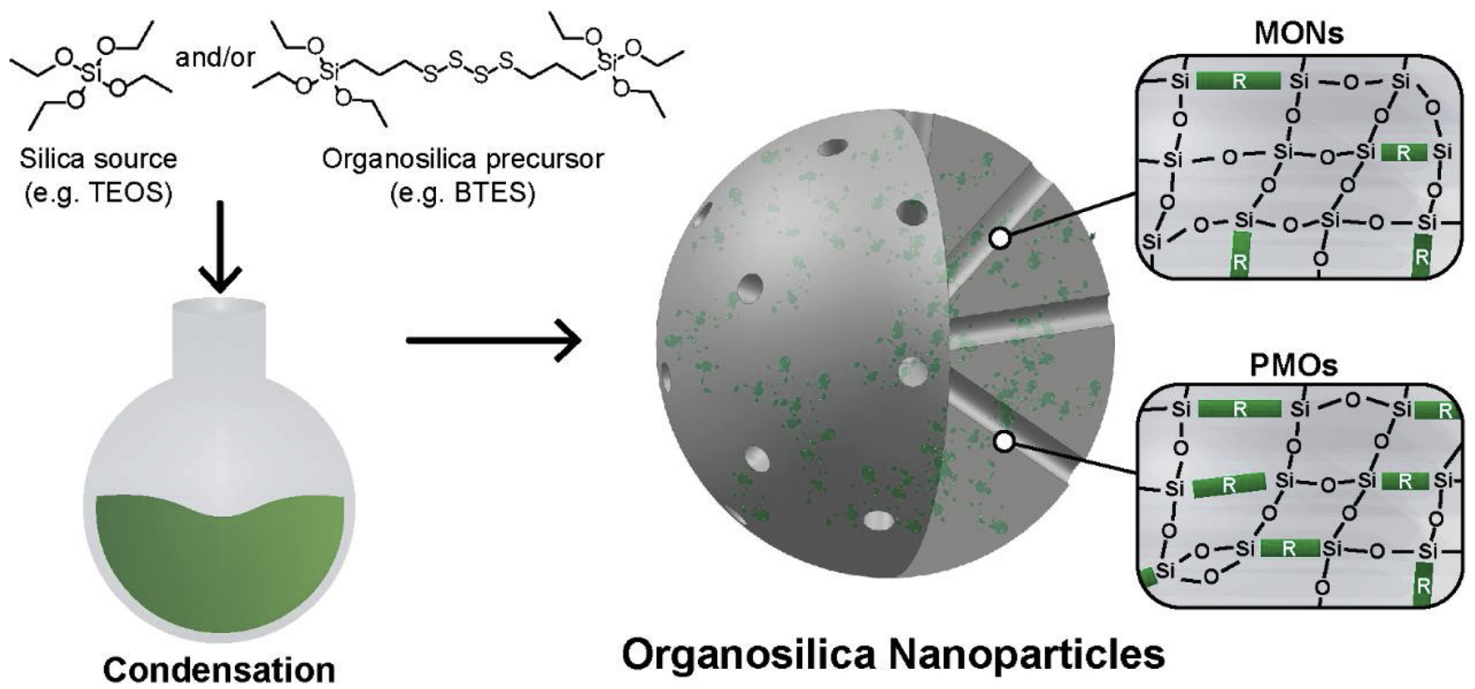

Fig. 1. Schematics of the organosilica nanoparticles' synthesis and matrix structure.

summarized (please see Table 2). The different organosilica precursors that can be used and the underlying principles that regulate the therapeutics release are described [66].

\section{1. pH-responsive organosilica nanoparticles}

The development of $\mathrm{pH}$-responsive nanocarriers for promoting a tumor-specific drug release is one of the most explored in cancer nanomedicine [71,72]. This approach aims to take advantage of the natural $\mathrm{pH}$ gradient that the nanoparticles will encounter when circulating in the human body [21]. During the nanoparticles' circulation in the bloodstream, they will be exposed to a neutral $\mathrm{pH}$ value $(\sim 7.4)$, however when reaching the tumor tissue the $\mathrm{pH}$ decreases to acidic values (around to 6.5) [47,73,74]. Such difference is attributed to the limited availability of oxygen and nutrients that favor the lactate production (i.e. Warburg effect) and consequent acidification of the extracellular tumor media [73,75]. Moreover, after the cancer cells internalization, the nanoparticles are exposed to even more acidic media in endosomal ( $\mathrm{pH}$ 5.5-6.0) and lysosomal ( $\mathrm{pH} 4.5-5.0)$ compartments $[47,76,77]$. Liu and colleagues developed doxorubicin(DOX)-loaded MONs with benzoic-imine bonds that confer them a $\mathrm{pH}$-responsive degradability and drug release [78].The organosilica nanoparticles were produced by promoting the co-condensation of a phenylene Schiff base bridged organosilane precursor and tetraethyl orthosilicate (TEOS) through a base-catalyzed modified-Stöber method. Additionally, the MONs' pores were also blocked by functionalizing their surface with PEGylated $\beta$-cyclodextrins. The authors observed that the incubation in PBS at pH 7.4 or culture medium supplemented with FBS (10\%) for 24 $\mathrm{h}$ did not induce any significant changes on the nanoparticles size and surface morphology. On the other side, the incubation in PBS (phosphate buffered saline) at pH 5.4 for only $8 \mathrm{~h}$ resulted in the reduction of the nanoparticles' size from 57.8 to $8 \mathrm{~nm}$, which was attributed to the hydrolysis of the schiff base organic bridge in acidic media, that lead to nanoparticle disassembly. Moreover, the authors also noticed that this pH-sensitivity could modulate the DOX release, $10 \%, 71 \%$, and $100 \%$ of drug release at $\mathrm{pH} 7.4,5.9$, and 5.4, respectively. In the in vivo studies, the authors verified that compared to the commercially available Doxil the MONs pH sensitivity could decrease the DOX systemic toxicity (i.e. accumulation in the major organs) and improve the antitumoral effect, final tumor volume of 102.38 and $190.47 \mathrm{~mm}^{3}$ for MONs and Doxil, respectively. In turn, Wu et al. explored the production of $\mathrm{pH}$ responsive hollow phenylene (aromatic)-bridged PMOs for delivering DOX to cancer cells [35]. The hollow PMOs were produced through a salt-assisted acid etching methodology using 1,4-bis(triethoxysilyl) benzene (BTEB) as the organosilica precursor. The authors observed that the DOX release occurs with stable and sustained profile at physiological pH, reaching the $8 \%$ in $24 \mathrm{~h}$. However, the replacement of the release media for a $\mathrm{pH} 4$ buffer solution prompted the drug release, reaching the $42.4 \%$ in the following $24 \mathrm{~h}$. This behavior is attributed to the establishment of drug/nanoparticle phenylene bridges interactions (e.g. $\pi-\pi$ stacking), which decreases with the $\mathrm{pH}$ acidification favoring the drug diffusion. Moreover, the authors observed that DOX delivery mediated by hollow phenylene (aromatic)-bridged PMOs could improve the cytotoxicity against DOX resistant MCF-7 (cancer cells, $90 \%$ and 70 $\%$ of cell viability for free drug and PMOs treated groups, respectively). Similarly, Moorthy and coworkers produced $\mathrm{pH}$ responsive MONs for mediating the dual-drug delivery to breast cancer cells [79]. In their approach, an organosilane precursor was produced by reacting 2,6-diaminopyridine with triethoxy(3-isocyanatopropyl)silane originating a bissilylated pyridine bridged diurea derivative. Then, the MONs were produced by inducing the co-condensation of the synthesized organosilane precursor and TEOS under acidic conditions and using Pluronic P123 as pore structuring agent. The authors observed that the MONs drug loading could be achieved through the establishment of hydrogen bounds and electrostatic interactions with the organosilane bridges in the nanoparticle matrix. Further, the authors noticed that these interactions conferred to the MONs a $\mathrm{pH}$ responsive drug release profile. In fact, the 5-fluorouracil presented a sustained release in physiological-like medium (10\% of drug released at $\mathrm{pH} 7.4)$ that was accelerated in acidic $\mathrm{pH}$ ( $87 \%$ drug released at $\mathrm{pH}$ 5.5). This sharp difference on the release profile was attributed to the protonation state of the pyridyle groups on the organosilane bridges, which at $\mathrm{pH}$ 7.4 allow the establishment of hydrogen bonds with 5 -fluorouracil that are lost with the pyridyle groups protonation in acidic $\mathrm{pH}$, occurring a strong electrostatic repulsion that favor the drug release. In the in vitro cancer cell cultures, the authors observed only a small decrease in the MCF-7 cells viability ( $10 \%$ of dead cells) when the MONs where administered with culture media at $\mathrm{pH}$ 7.4. Nevertheless, the MONs administration under acidic media ( $\mathrm{pH}$ 6.0) decrease the cancer cells viability in $\approx 90 \%$.

Apart from the utilization of organosilane bridge to mediate the nanoparticles' pH-responsivity, the controlled drug release profile on organosilica nanoparticles can also be controlled by pore blocking moieties grafted on its surface. Parambadath et al. created a pH-responsive organosilica nanoparticle by blocking the pore openings with $\mathrm{N}$-[3-(trimethoxysilyl)propyl]aniline/ $\beta$-cyclodextrin ( $\beta$-CD) nanovalves [80]. The MONs were produced by promoting the co-condensation of $\mathrm{N}^{\prime}$-bis[3-(triethoxysilyl)propyl] ethyl-enediamine (TESEN) and 


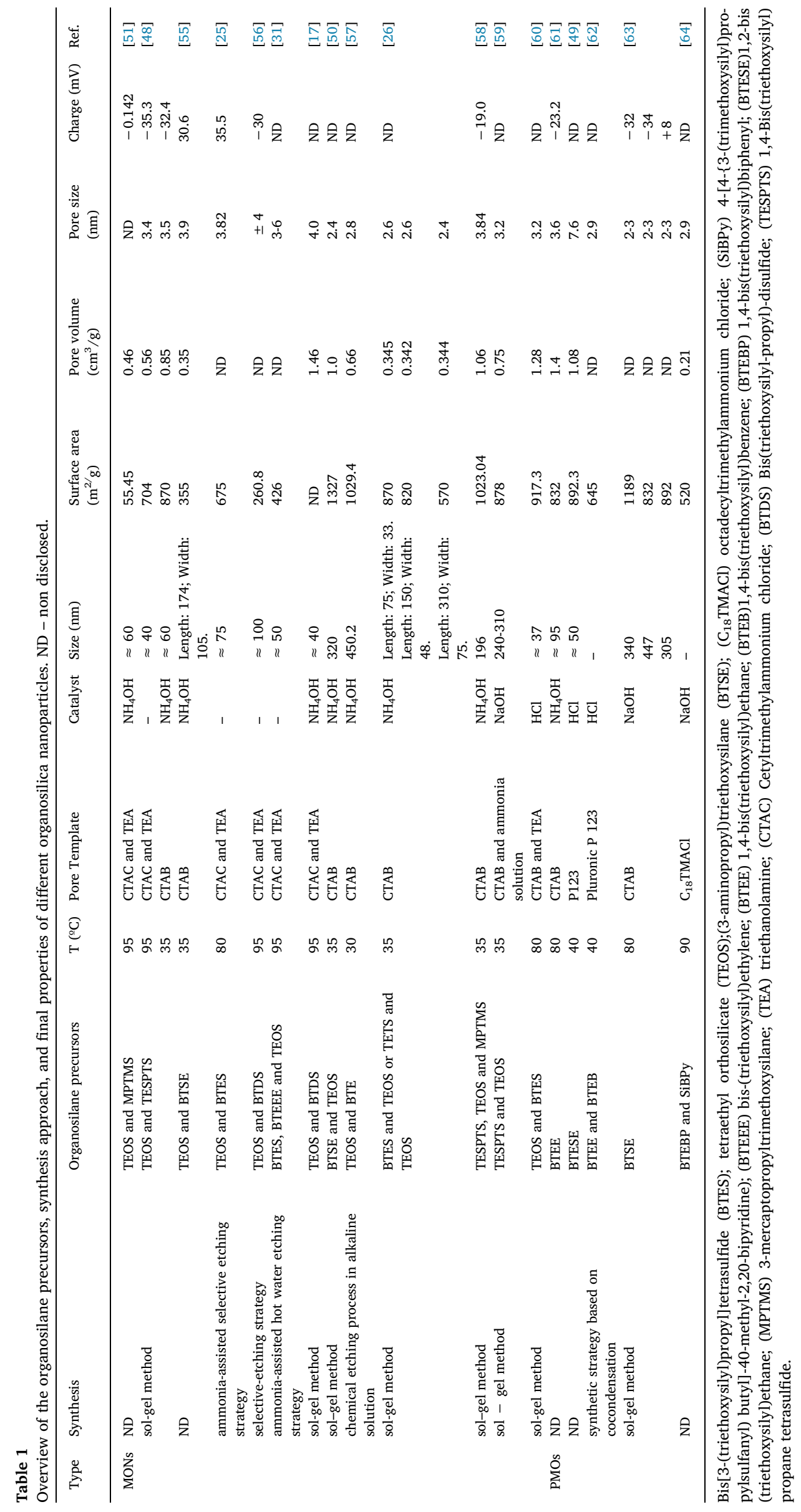




\section{Organosilica Nanoparticles}

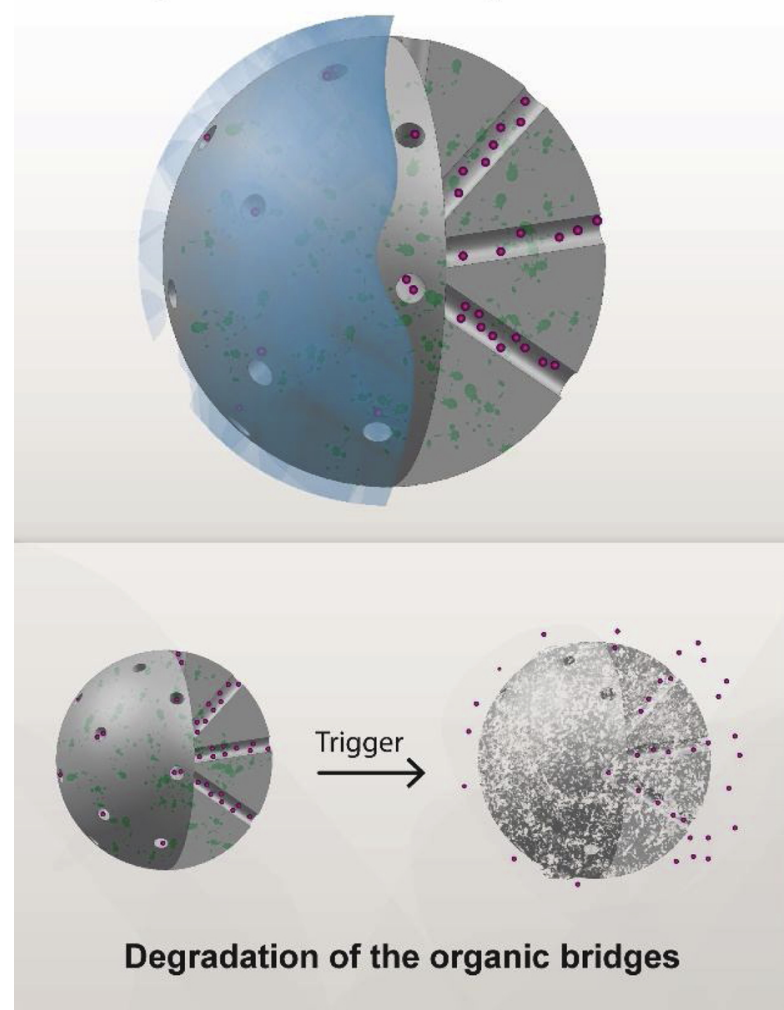

\section{Common Triggers}

$\mathrm{pH}$

Redox potential

Enzymes

NIR laser

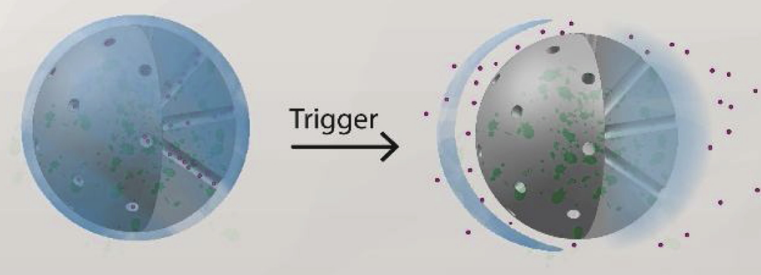

Removal or degradation of the pore caps

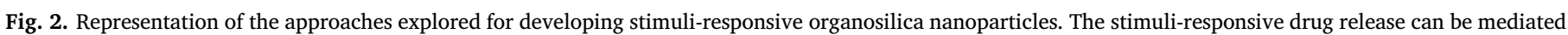
by the degradation of the organic bridges present on the nanoparticle matrix or through the utilization of pore capping agents.

tetramethyl orthosilicate (TMOS) under basic conditions and using CTAB as pore structuring agent. Then, the MONs surface was modified with N-[3-(trimethoxysilyl)propyl]aniline and then, after the drug loading, functionalized with $\beta$-CD through host-guest interactions. The authors observed that the $\mathrm{pH}$ responsive nanovalves could prevent the drug leakage at $\mathrm{pH} 7.4,1 \%$ of the drug released. However, the MONs incubation in acidic medium $(\mathrm{pH} 4)$ resulted in the release of $80 \%$ of 5fluorouracil, which is justified by TESEN protonation and consequent disassembly of the $\beta$-CD nanovalves (Fig. 3).

$\mathrm{Hu}$ and colleagues also explored the MONs surface functionalization to develop a $\mathrm{pH}$ responsive antitumoral drug delivery system [47]. In their approach, MONs were produced through the co-condensation of TEOS and bis[3-(triethoxysilyl)propyl]tetrasulfide (BTES) under basic conditions and using cetyltrimethylammonium chloride as pore structuring agent. Afterwards, the MONs were functionalized with polyacrylic acid and glutathione (GSH) for controlling the drug release and enhance the cells internalization, respectively. The authors observed that the amount of DOX released increased with the acidification of the release medium, $7 \%, 16.1 \%$, and $45.5 \%$ at $\mathrm{pH} 7.4,6.5$, and 5 , respectively. This behavior is mediated by the protonation of the GSH grafted polyacrylic acid chains, decreasing the electrostatic interaction with the DOX and allowing its diffusion to the outer medium. Furthermore, the authors observed in the in vitro assays that the conjugation of the GSH-mediated enhanced cellular uptake with the MONs pH-responsive drug delivery could reduce the HeLa cancer cells viability in $80 \%$ using low nanoparticle concentrations $(10 \mu \mathrm{g} / \mathrm{mL})$.

\subsection{Redox-triggered release}

The redox potential is also one of the most explored stimuli-triggers that have been explored to create drug delivery nanosystems with tailored release profiles $[81,82]$. The drug release from redox responsive nanoparticles is usually mediated by the degradation of disulfide bonds (i.e. R-S-S-R) in the presence of reducing agents [83]. For this purpose, the GSH/glutathione disulfide pair is one of the most important antioxidant defense mechanisms in the human body and its expression in the cancer cells is 3 orders in magnitude superior to that of the healthy tissues [84]. Taking this into account, Moghaddam and colleagues developed redox-responsive MONs for delivering anticancer chemotherapeutics [37]. The authors produced two different redox-responsive MONs by promoting the co-condensation of TEOS and bis(triethoxysilyl-propyl)-disulfide (BTDS) (disulfide-based MONs) or TEOS and BTES (tetrasulfide-based MONs) under a basic environment in the presence of CTAB. The authors reported that the resulting disulfidebased and tetradisulfide-based MONs presented a similar size $(\sim 110$ $\mathrm{nm})$, surface charge $(-32$ and $-27 \mathrm{mV}$ ) and pore diameters (2 and 2.7 $\mathrm{nm})$. Nevertheless, the in vitro degradation studies performed in the GSH presence revealed that the disulfide-based MONs have a higher degradation rate, reaching the $14 \%$ in 15 days. Further, the authors also observed that in the absence of GSH the DOX is released in a sustained fashion from the MONs reaching the $40 \%$ after 15 days, whereas the addition of GSH can promote a faster DOX release ( $>50 \%$ in 15 days) due to the particles' degradation. Similarly, Yue et al. developed MONs containing disulfide bridges to deliver DOX to liver cancer cells [85]. For that purpose, TEOS and BTES were reacted under basic conditions in the presence of CTAB. The authors observed that the resulting MONs were completely degraded after $72 \mathrm{~h}$ in PBS containing $10 \mathrm{mM}$ of GSH, in a process mediated by the disruption of the nanoparticle disulfide bonds through reduction reactions. Moreover, the authors also demonstrated that the DOX release could be accelerated in 


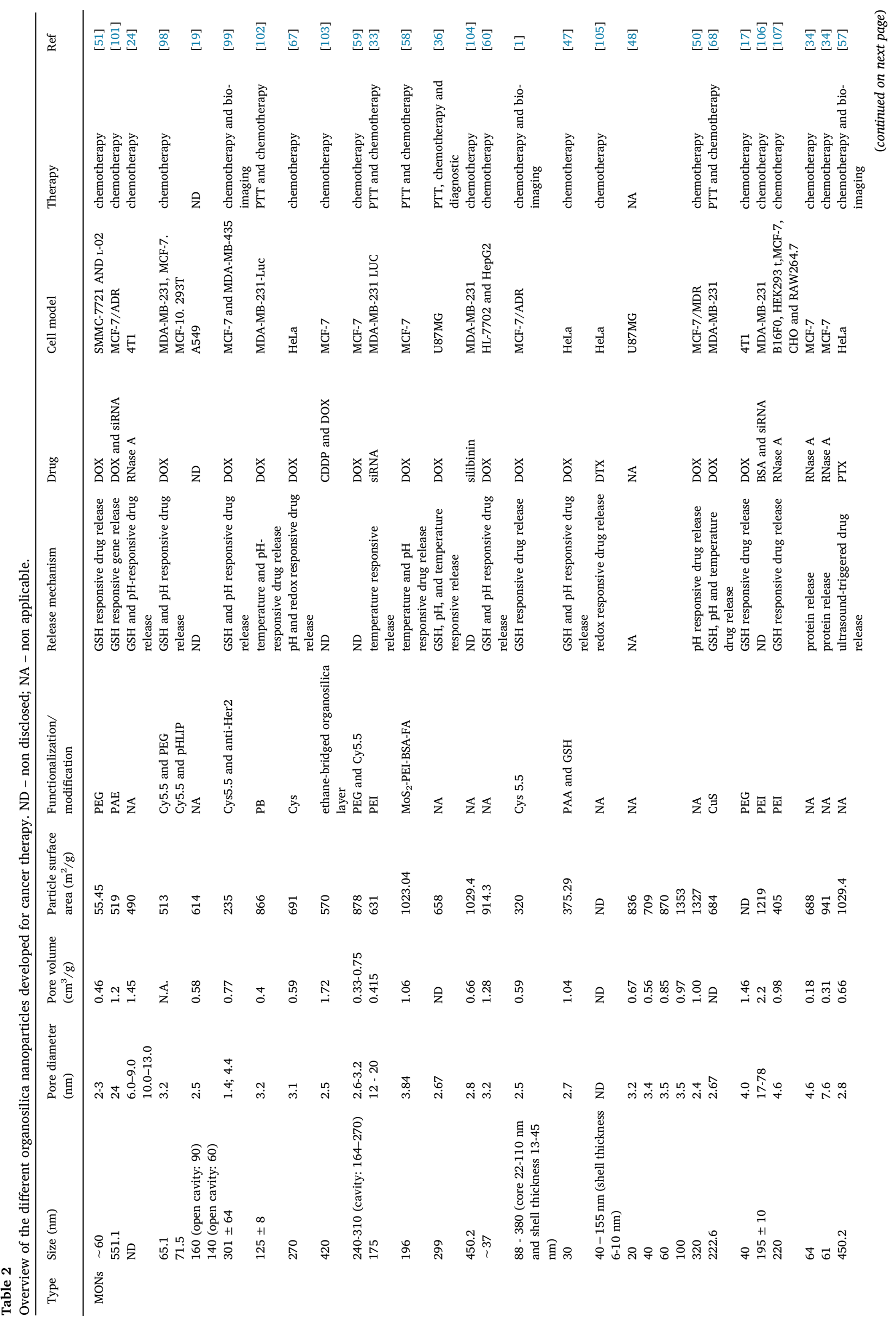




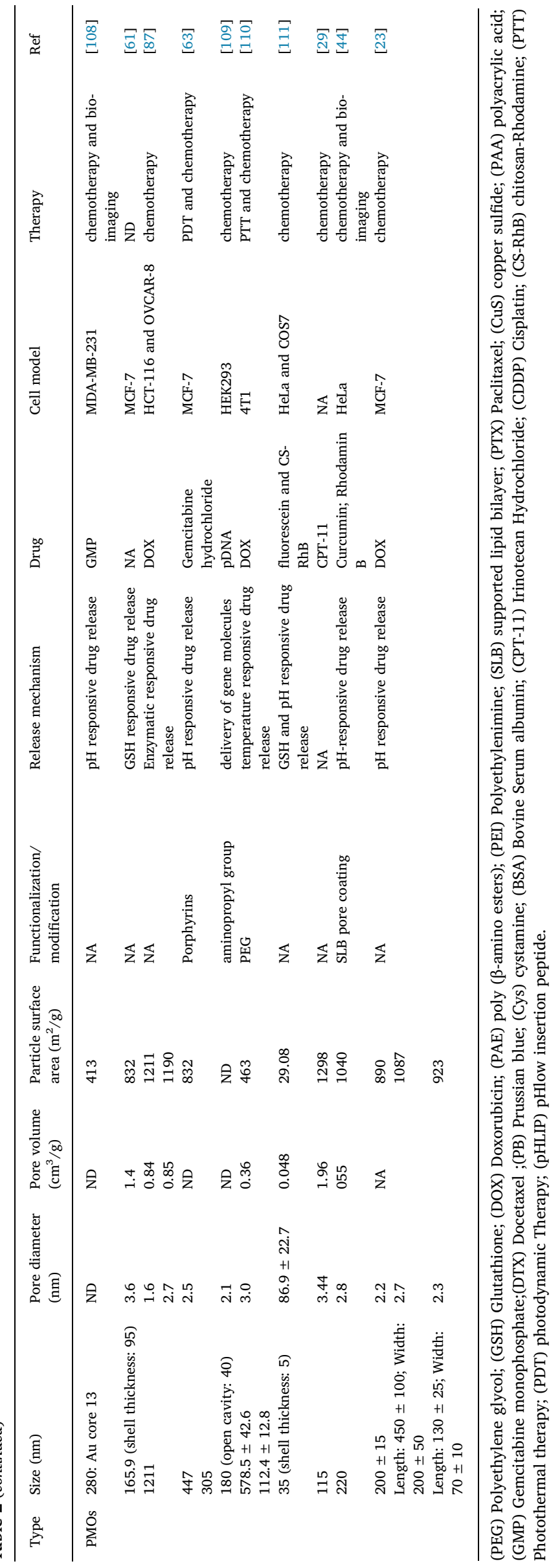

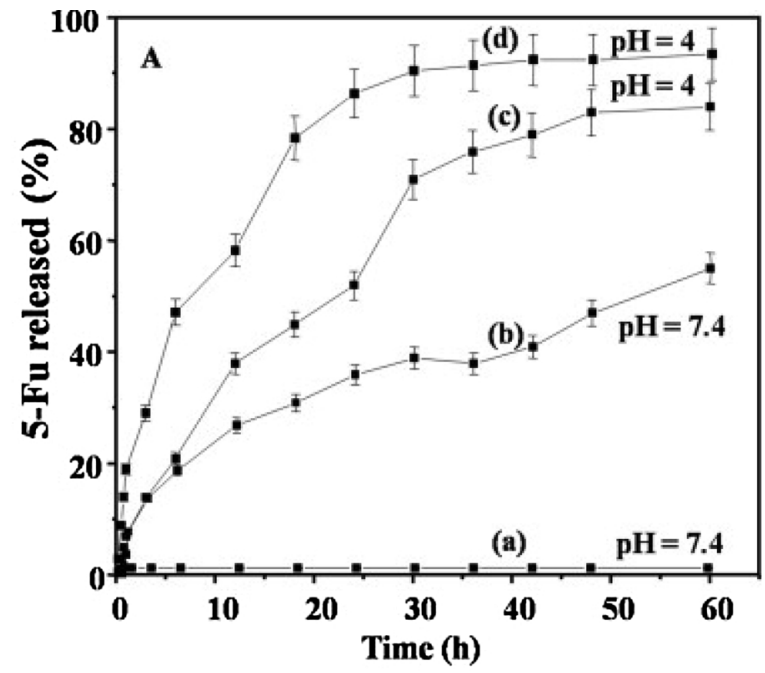

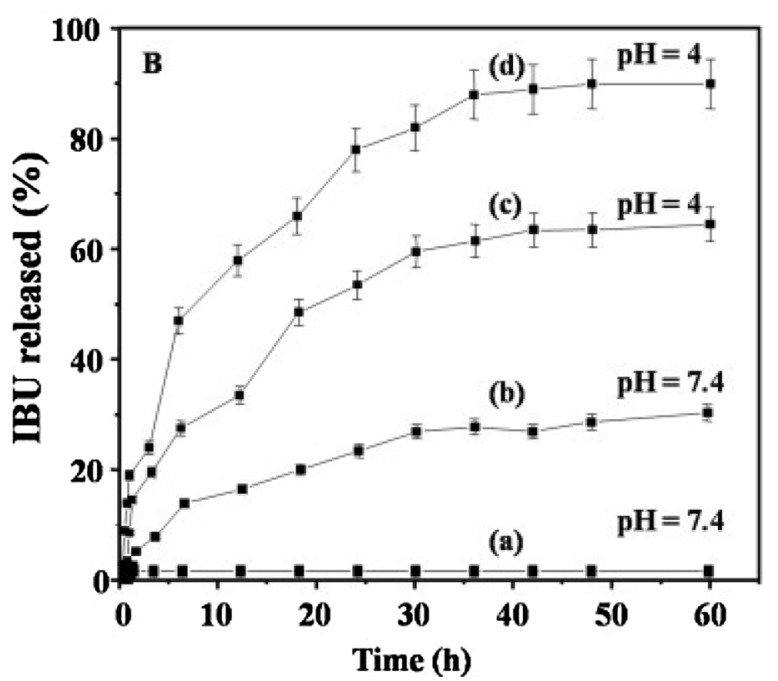

Fig. 3. Analysis of the 5-fluorouracil (5-Fu) and ibuprofen (IBU) release at different $\mathrm{pH}$ from $\beta$-CD capped MONs (a) and (c) and non-capped MONs (b) and (d). Reprinted from Microporous and Mesoporous Materials, Vol. 215, Parambadath, S., Mathew, A., Barnabas, M.J., and Ha, C.S., A pH-responsive drug delivery system based on ethylenediamine bridged periodic mesoporous organosilica, Pages No.67-75, Copyright (2015), with permission from Elsevier.

the presence of GSH, $<5 \%$ and $30 \%$ of drug released after $24 \mathrm{~h}$ at $\mathrm{pH}$ 7.4 in the absence or presence of GSH, respectively. This behavior also increased the intracellular DOX concentration in HepG2 cancer cells. Additionally, the superior control over the drug release resulted in an enhanced antitumoral effect against HepG2 cells when comparing to conventional mesoporous silica nanoparticles. Huang and colleagues developed polyethylene glycol functionalized hollow MONS for the redox responsive DOX release to 4T1 breast cancer tumors [17]. The hollow PMOs were produced through an etching methodology using TEOS and BTDS as silica precursors for producing the organosilica shell. Subsequently, a condensation reaction between the hollow MONs and Methoxy Polyethylene glycol (PEG) silane was performed to functionalize the particles' surface. The authors observed that the hollow MONs incubation on simulated body fluid containing GSH (10 $\mathrm{mM}$ ) for 2 days induced a significant degradation on the particles structure, which were completely degraded in 14 days, whereas in the absence of GSH the particles remained stable for about 11 days. This biodegradation capacity was observed in Transmission Electron Microscopy (TEM) after the nanoparticles' uptake by 4T1 cancer cells, with the majority of the nanoparticles collapsing after 2 days and very few particles remained visible after 7 days (Fig. 4). Such behavior 

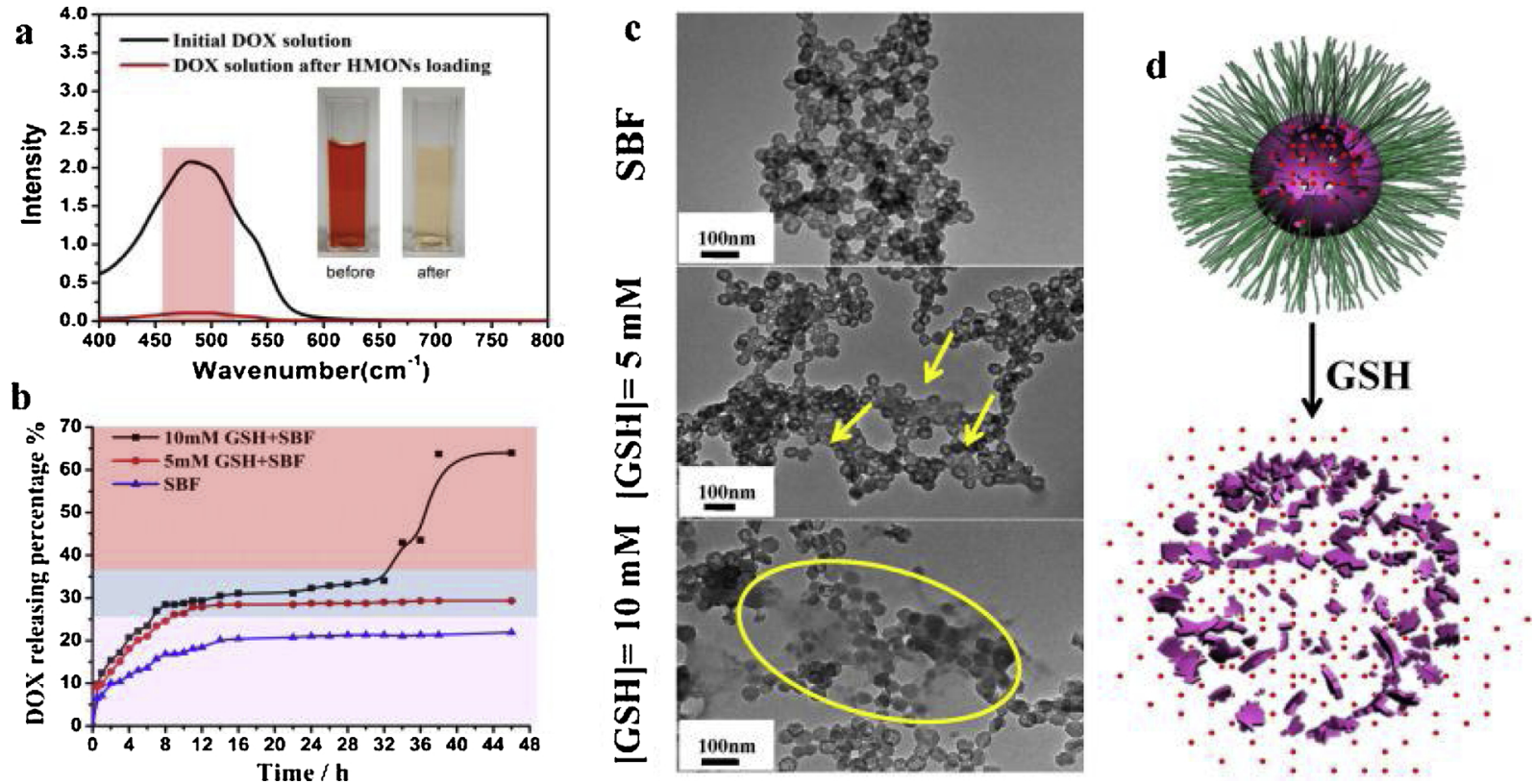

Fig. 4. Evaluation of the DOX loading and release from polyethylene glycol functionalized hollow MONS. (A) UV-vis spectra of the DOX solution before and after the encapsulation procedure. (B) Analysis of the DOX release and (C) TEM images of polyethylene glycol functionalized hollow MONS in the presence or absence of GSH. (D) Schematics of the MONs degradation in response to the GSH. Reprinted from Biomaterials, Vol 125, Huang, P., Chen, Y., Lin, H., Yu, L., Zhang, L., Wang, L. Zhu, Y., and Shi, J., Molecularly organic/inorganic hybrid hollow mesoporous organosilica nanocapsules with tumor-specific biodegradability and enhanced chemotherapeutic functionality, Pages No.23-37, Copyright (2017), with permission from Elsevier.

demonstrates the importance of the incorporation of disulfide bridges on the organic phase of the MONs matrix. Furthermore, the authors promoted the DOX loading by exploiting hydrophobic interactions between the drug and particle structure and observed that the hollow MONs redox-responsive degradation enhanced the drug release. In fact, the redox responsiveness was clear after $48 \mathrm{~h}$ of incubation with $60 \%$, $30 \%$ and $20 \%$ of drug released with GSH $20 \mathrm{mM}, 10 \mathrm{mM}$, and $0 \mathrm{mM}$. In the in vivo assays, the redox-responsive PEGylated hollow MONs were able to impair the tumor progression more efficiently than free DOX, tumor-inhibition percentage of $72.2 \%$ and $37.5 \%$ for MONs and free drug, respectively.

Alternatively, Shen and coworkers produced a pore-capped redoxresponsive MONs for delivering DOX to hepatocarcinoma SMMC-7721 tumors [51]. In this approach, an organosilica layer based on 3-mercaptopropyl-trimethoxysilane was grown on the surface of mesoporous silica nucleus and then reacted with maleimide-polyethylene glycol for blocking the drug release. The authors observed that the MONs incubation in PBS containing GSH $(10 \mathrm{mM})$ induces a layer-based degradation characterized by the initial removal of the PEG superficial layer followed by the dissociation/degradation of the disulfide-doped organosilica outer coating. Further, this GSH mediated degradation could also modulate the DOX release. In fact, the authors registered an increased DOX release by raising the GSH concentration, $40 \%$ and 72 $\%$ of DOX released after $48 \mathrm{~h}$ in PBS at pH 7.4 with $2 \mathrm{mM}$ and $10 \mathrm{mM}$ $\mathrm{GSH}$, respectively. This increased control over the DOX release demonstrated to be advantageous on the in vitro cytotoxicity studies. The authors observed that the MONs (DOX concentration $50 \mu \mathrm{g} / \mathrm{mL}$ ) induced the reduction of SMMC-7721 tumor cells viability to $20 \%$, whereas in L-02 normal cells only a reduction to $90 \%$ of cell viability was observed. In the in vivo assays, the PEGylated MONs induced a tumor inhibition rate of $71.3 \%$ (free DOX tumor inhibition rate 36.2 $\%$, without inducing significant changes on the rat body weight or damage to major organs.

\subsection{Other stimuli-responsive organosilica nanoparticles}

Despite the most common $\mathrm{pH}$ - or redox- responsive organosilica nanoparticles, there are others that release the drugs in response to other stimuli such as temperature, enzymes, and ultrasounds. For example, Shao and colleagues developed near-infrared (NIR)-responsive PEGylated MONs doped with $\mathrm{MoS}_{2}$ nanosheets to be used in the chemophotothermal therapy of breast cancer [86]. For that purpose, thioether bridged MONs were produced by promoting the co-condensation of TEOS and 1,4-bis(triethoxysilyl) propane tetrasulfide (TESPTS) under basic conditions in the presence of CTAB. Then, the particles surface was modified with 3-aminopropyltrimethoxysilane and incubated with $\mathrm{MoS}_{2}$ nanosheets and maleimide-polyethylene glycol. The obtained nanoparticles presented the DOX encapsulated in the mesopores and its diffusion was blocked by the $\mathrm{MoS}_{2} / \mathrm{PEG}$ coating. The authors observed that the $\mathrm{MoS}_{2} / \mathrm{PEG}$ MONs $(1 \mathrm{mg} / \mathrm{mL})$ could mediate a temperature increase up to $50{ }^{\circ} \mathrm{C}$ under NIR laser irradiation $\left(808 \mathrm{~nm}, 1 \mathrm{~W} / \mathrm{cm}^{2}\right.$ for 5 $\mathrm{min}$ ), which could be explored to accelerate the drug release. In fact, in the absence of the NIR laser, less than $1 \%$ of the DOX is released in $1 \mathrm{~h}$, whereas under NIR irradiation increased to $16 \%$. Such was attributed to the heat generation and consequent vibration of $\mathrm{MoS}_{2}$ sheets at the surface of the MONs that decrease the drug/nanoparticle interactions and to the increased movement of the DOX molecules. Further, the authors also observed an increased amount of DOX in the interior of Huh-7 cells after NIR laser irradiation. In the in vitro cytotoxicity studies, the authors observed that the $\mathrm{MoS}_{2} / \mathrm{PEG}$ MONs combination of the controlled DOX delivery with the heat generation (photothermal effect) could reduce the MCF-7 cells viability to $15 \%$, whereas the stand-alone chemotherapeutic or photothermal treatments mediated by the MONs presented a cellular viability of $49 \%$ and $65 \%$, respectively.

On the other hand, researchers can also utilize enzymes, that are overexpressed in the tumor tissues, to trigger the drug release from the organosilica nanoparticles. In this way, Omar et al. produced enzymatic responsive PMOs for the DOX delivery to cancer cells [87]. The authors synthesized two different PMOs by promoting the co-condensation of an diazobenzene-triethoxysilyl amide with 1,4-bis-(triethoxysilyl)benzene (AZO-E PMOs) or 1,2-bis-(trimethoxysilyl)ethane (AZO-B PMOs) under basic conditions in the presence of $\mathrm{CTAB}$. The authors observed that the AZO-B PMOs have a faster degradation profile in the presence of azoreductase, which translated to a faster drug release, $\sim 100 \%$ and 
$\sim 80 \%$ of DOX was released, after $48 \mathrm{~h}$ of incubation with enzyme for AZO-E and AZO-B PMOs, respectively. In contrast, the AZO-E PMOs present a superior biocompatibility, $95 \%$ and $60 \%$ of cell viability at a concentration of $100 \mu \mathrm{g} / \mathrm{mL}$ for AZO-E and AZO-B PMOs, respectively. Moreover, the authors reported a noticeable decrease in chicken egg OVCAR-8 tumors size after the administration of the enzyme responsive PMOs.

Another example of the organosilica nanoparticles plasticity is the capacity to produce drug delivery systems that are responsive to multiple stimulus, allowing to enhance the spatial-temporal control of the cargo release. In this field, one of the most common approaches is to combine the $\mathrm{pH}$ and redox sensitivity to modulate the drug release. Rao and colleagues produced a cystamine (Cys)-integrated MON for the dual-stimuli responsive delivery of DOX to HeLa cancer cells [67]. For that purpose, the authors synthesized a Cys containing organosilica precursor by reacting 3-(Triethoxysilyl)propylsuccinic anhydride with Cys hydrochloride. Then, the organosilica precursor was co-condensed with TEOS under basic condition in the presence of CTAB. The incorporation of the organic bridge increases the interaction of the DOX with the nanoparticle matrix improving the drug loading though the complexation of the carboxylic groups on the Cys-integrated MON and the amino groups of DOX. Moreover, the authors observed that the DOX release was increased in the presence of acidic ( $\mathrm{pH} 5.5)$ and redox $(1,4-$ dithiothreitol (DTT) $10 \mathrm{mM}$ ) conditions due to the $\mathrm{pH}$ cleavage and $\mathrm{S}-\mathrm{S}$ bonds degradation. In fact, the amount of DOX released increased from $10 \%$ at $\mathrm{pH} 7.4-56 \%$ and $89 \%$ with the nanoparticles' incubation at $\mathrm{pH}$ 5.5 and $\mathrm{pH} 5.5 / 10 \mathrm{mM}$ DTT for $48 \mathrm{~h}$, respectively. In addition, the authors observed that this behavior influenced the Cys-integrated MONs cytotoxicity towards HeLa cancer cells. The MONs incubation with the cancer cells at physiological conditions $(\mathrm{pH} 7.4)$ resulted in a reduction of the cell viability to $83 \%$, whereas in acidic conditions $(\mathrm{pH}$ 5.5) or acidic redox condition ( $\mathrm{pH} 5.5+10 \mathrm{mM} \mathrm{GSH}$ ) the MONs reduced the HeLa cells viability to $13 \%$ and 5\%, respectively. Similarly, Ren and coworkers developed a $\mathrm{pH}$ - and redox- responsive nanoparticle

(A)
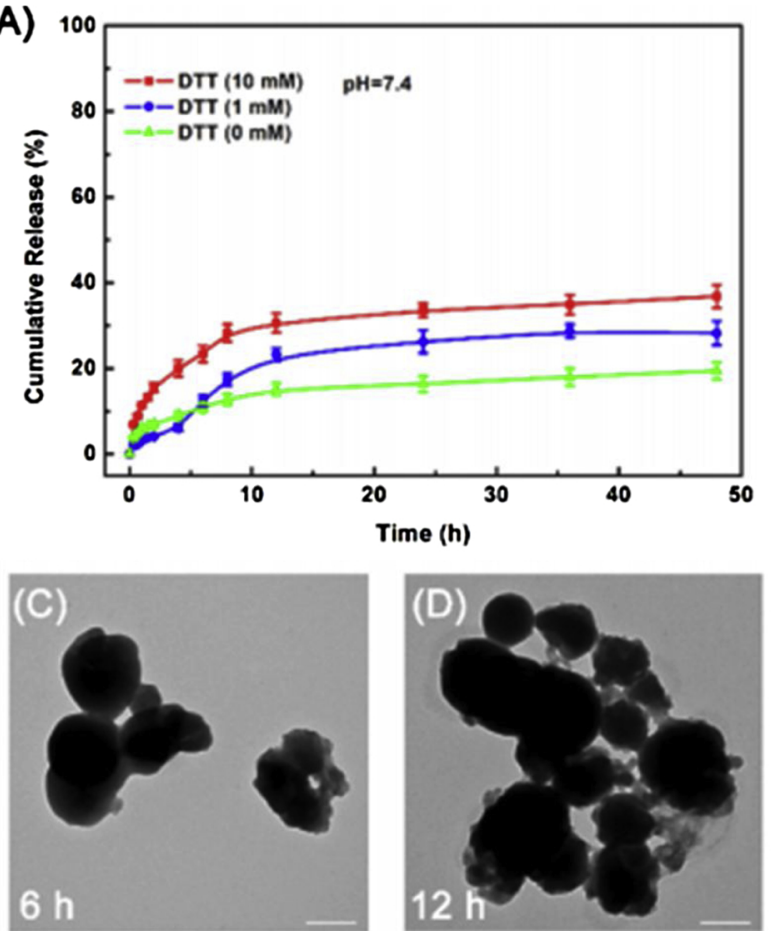

based on a ZIF-8 core and a mesoporous organosilica shell for delivering DOX to HeLa cancer cells [88]. To accomplish that, the organosilica coating layer was produced through the co-condensation of TEOS and BTDS under basic conditions in the presence of DOX loaded ZIF-8 cores. The authors observed that the DOX release could be accelerated by the acidification of the pH, $20 \%$ and $40 \%$ of DOX released in $48 \mathrm{~h}$ at $\mathrm{pH}$ 7.4 and 5, respectively. Additionally, the authors also demonstrated that the addition of DTT $1 \mathrm{mM}$ or $10 \mathrm{mM}$ could further increase the DOX release to $27 \%$ and $38 \%$ ( $48 \mathrm{~h}$ at $\mathrm{pH} 7.4$ ), respectively (Fig. 5). This behavior was attributed to the acid-dependent degradation of the ZIF-8 cores and to the degradation of the organosilica shell S-S bonds under reductive environments, as the authors observed in TEM images. In the in vivo studies, the authors observed that the nanoparticles induced a significant reduction of the tumor weight (inhibition rate of $89.4 \%$ ) being observed severe necrotic zones in the histological studies.

Alternatively, $\mathrm{Wu}$ et al. developed a thermo- and $\mathrm{pH}$-responsive MONs coated with $\mathrm{MoS}_{2}$ sheets for deliver DOX to breast cancer [58]. The MONs were produced through the co-condensation of TESPTS and TEOS under basic conditions in the presence of CTAB. Then, the MONs surface was modified with 3-mercaptopropyltrimethoxysilane and reacted with $\mathrm{MoS}_{2}$ sheets modified with polyethylenimine (PEI), bovine serum albumin (BSA), and folic acid. The authors observed that the nanoparticles could mediate an increase in the temperature to $45.8{ }^{\circ} \mathrm{C}$ after NIR ( $808 \mathrm{~nm}, 1 \mathrm{~W} / \mathrm{cm}^{2}$ for $5 \mathrm{~min}$ ) laser irradiation. Additionally, this photothermal capacity could increase the DOX release rate, $2 \%$ and $15 \%$ after $1 \mathrm{~h}$ in the absence or presence of NIR laser irradiation, due to the heat generation and vibration of the $\mathrm{MoS}_{2}$ nanosheets that facilitate the drug diffusion. The authors also observed a faster DOX release with the acidification of the media, $30 \%$ and $60 \%$ drug released, after $72 \mathrm{~h}$ at pH7.4 and 5, in the presence of NIR light irradiation. The controlled drug release resulted in an increased biosafety and longer survival periods in the in vivo assays combined with the inhibition of the MCF-7 tumors growth with the combinatorial chemophotothermal therapy. With a similar approach, Lu and coworkers
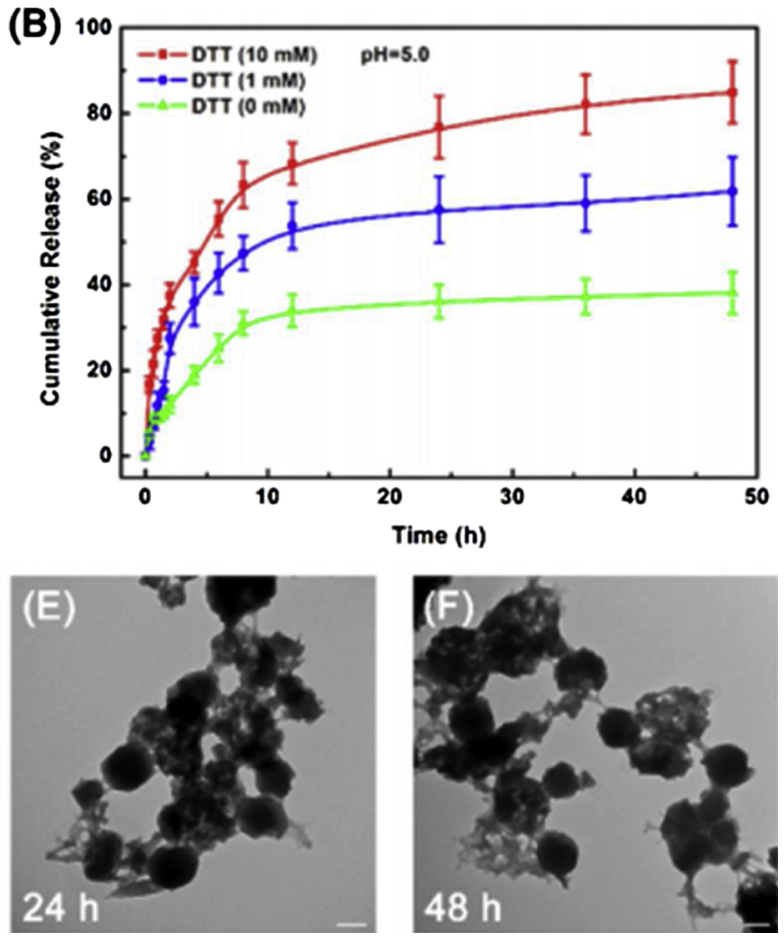

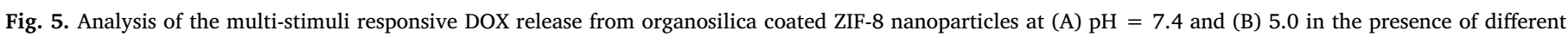

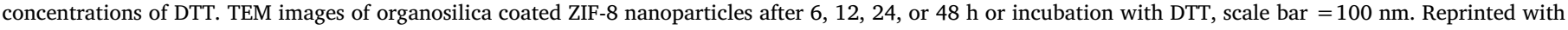

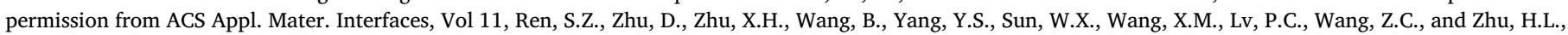

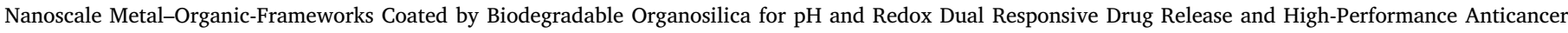
Therapy, Pages No. 20678-20688, Copyright (2019) American Chemical Society. 
created a triple-stimuli responsive copper sulfide doped MONs for the chemo-photothermal therapy of glioblastoma [36]. For that purpose, thiol-functionalized yolk-shell MONs were produced through an acidetching process based on the co-condensation of TESPTS and TEOS. Subsequently, the MONs were functionalized with 3-mercaptopropyltrimethoxysilane and doped with copper sulfide. The authors demonstrated that the copper sulfide doping conferred to the MONs photothermal capacity, reaching the $42{ }^{\circ} \mathrm{C}$ after the NIR laser irradiation (808 $\mathrm{nm}, 0.8 \mathrm{~W} / \mathrm{cm}^{2}$ for $3 \mathrm{~min}$ ). Furthermore, due to the disulfide bonds present on the organosilica matrix, the DOX release could be accelerated by the presence of GSH, $13 \%$ and $40 \%$ of drug released after $8 \mathrm{~h}$ in the absence and with GSH. Moreover, the authors also demonstrated that the presence of NIR light and acidic environment would also modulate the DOX diffusion, $40 \%-62.5 \%$ drug released in the presence of GSH at pH 7.4 and 5.5 and $10 \%-20 \%$ drug released at $\mathrm{pH}$ 7.4 without or with NIR laser irradiation. In the in vivo studies, the authors reported the inhibition of the tumor growth on the group treated with the copper sulfide doped MONs mediated chemo-photothermal therapy, contrasting with continuous tumor growth observed in the free drug and single therapy treated groups.

\subsection{Surface modifications performed to improve the organosilica-based nanoparticles accumulation on tumor tissue}

The specific delivery of the therapeutic agents to cancer cells is one of the main objectives in cancer nanomedicine. The nanoparticles accumulation on the tumor tissue can occur via passive or active targeting [89-91]. The passive targeting explores the defective vasculature of the tumor tissue in order to allow the nanoparticles accumulation in this region [92]. This can occur via the enhanced permeation and retention
(EPR) effect caused by the tumor vessels leaky epithelium and discontinuous microvasculature with fenestrations sizes of approximately $400 \mathrm{~nm}$ that allows the nanoparticles extravasation to the tumor $[2,93,94]$. Recently, it was also described the occurrence of temporary vascular eruptions that allow the diffusion of the blood vessel content to the tumor tissue [95]. In this way, the optimization of the nanoparticles' stability and blood circulation time is essential to increase the nanoparticles probability to explore this passive accumulation on the tumor. In fact, various studies already demonstrated that the adsorption of biomolecules (e.g. proteins) on the nanoparticles' surface is one of the most important phenomena impacting the biodistribution, cellular uptake, and toxicity of the nanomedicines [94,96]. Therefore, the researchers have been exploring the nanoparticles surface passivation (e.g. PEG, polyoxazolines, and self-materials) to increase the blood circulation time and consequently the tumor accumulation (Fig. 6) $[2,94,97]$.

The PEG can improve the nanomaterials hydrophilicity and stability as well as decrease the surface opsonization and consequent the recognition by the immune system, which can result in an improved accumulation in the tumor tissue [94]. Zhang and colleagues produced a DOX loaded MON modified with $\mathrm{pH}$ low insertion peptide (pHLIP)-PEG for the breast cancer therapy [98]. The introduction of the pHLIP-PEG on the particles surface was achieved by reacting thiol groups on MONs with the maleimide group on PEG. The authors reported an increase in the nanoparticles size from $60 \mathrm{~nm}$ to $80 \mathrm{~nm}$ and a neutralization of the surface charge from $-13.96 \mathrm{mV}$ to $-11.19 \mathrm{mV}$ with the inclusion of the pHLIP-PEG moieties. Additionally, the authors did not observe any negative effect on the rats weight and major organs after the weekly administration of pHLIP-PEG MONs for more than 30 days. Similarly, Huang et al. developed a PEGylated MONs for the chemotherapy of

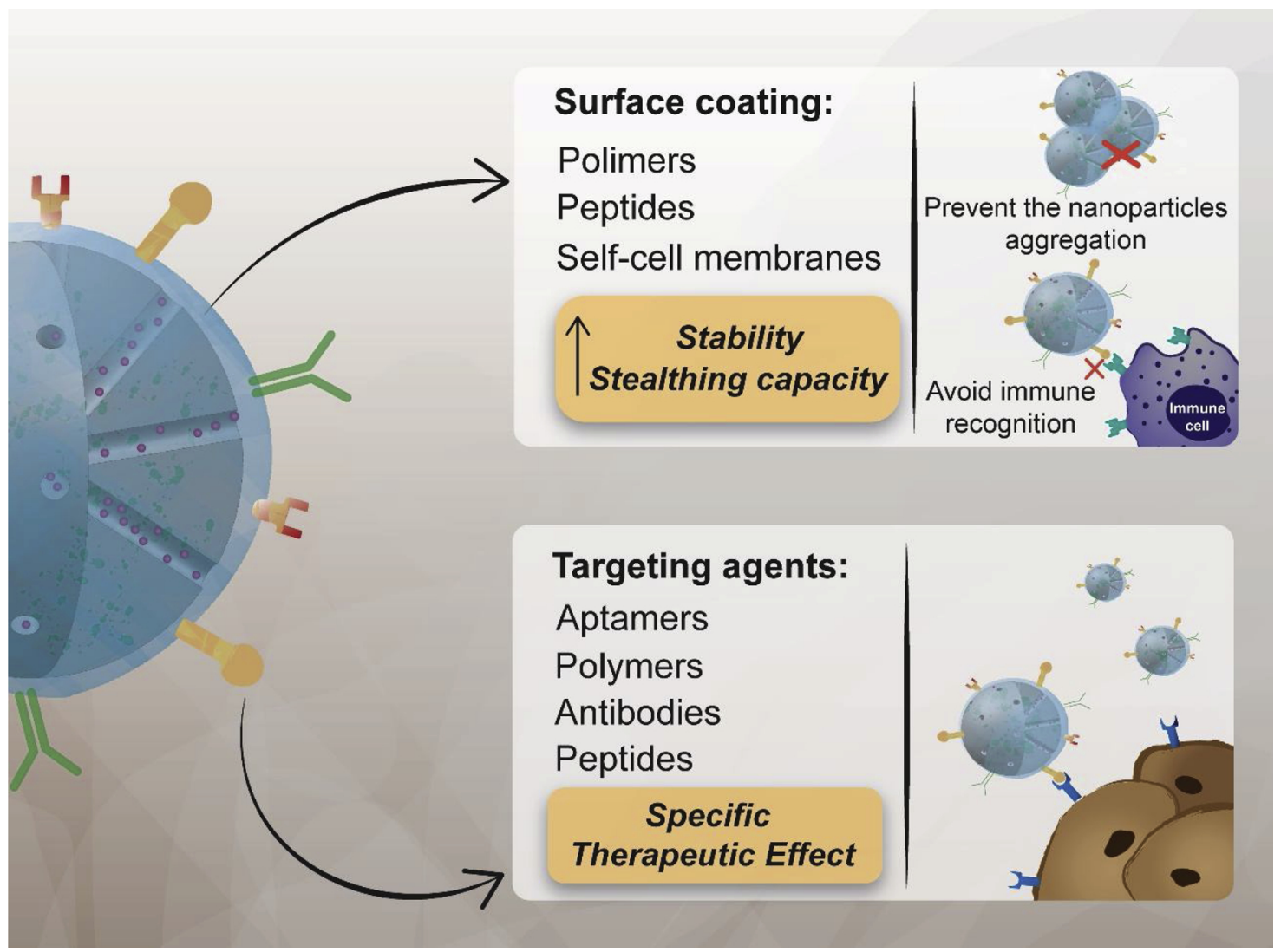

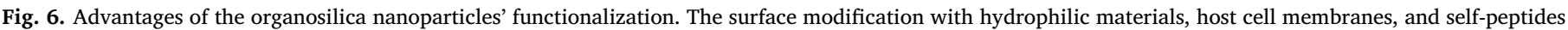

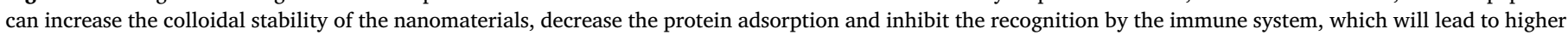

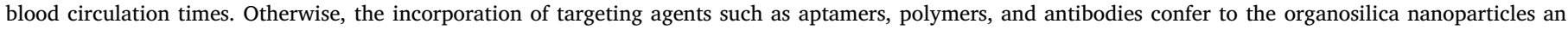
increased specificity towards the target cells or tissue. 
breast cancer [17]. The PEG moieties were introduced on the nanoparticles by promoting the condensation of a methoxy PEG silane in the MONs surface. In the in vivo studies, no changes were noticed on the rats weight, serum biochemical levels (e.g. alanine aminotransferase, blood urea nitrogen and creatinine), and blood components (e.g. mean corpuscular hemoglobin, white blood count, red blood count, and hemoglobin), demonstrating the PEGylated MONs biocompatibility.

On the other hand, the nanoparticles specific accumulation on tumor tissue can be stimulated by using targeting moieties that will promote ligand-receptor, antigen-antibody, and other forms of molecular recognition with the tumor tissue (e.g. cancer cells, tumor vasculature, and stromal constituents) [91]. Lu and coworkers developed an anti- Her2 affibody targeted PEGylated MON doped with Cy5.5 for the therapy and imaging of breast cancer [99]. The yolk-shell MONs were synthesized by promoting the co-condensation of TEOS and TESPTS, under basic conditions in the presence of CTAB, followed by an hydrothermal treatment. Then Cy5.5 maleimide and Her2 affibodyPEG-maleimide were reacted with the MONs and selectively conjugated in the outer surface through click chemistry of thiol and maleimide groups. The Her2 is a transmembrane tyrosine kinase receptor that has been recognized as a major classifier of invasive breast cancer, which can be used to direct the nanomedicines for these tumors. In fact, the studies performed in MDA-MB-435 (cell line overexpressing the Her2) and MCF-7 (cell line without Her2 receptor) cancer cells revealed that the Her2 affibody targeted MONs have an increased cellular internalization in MDA-MB-435 cancer cells. On the other hand, the authors observed an unspecific cellular internalization (i.e. similar uptake both on MDA-MB-435 and MCF-7 cells) in the groups treated with the nontargeted formulations. Moreover, they also reported that this improved selectivity resulted in a superior cytotoxicity towards MDA-MB435 cells, $60 \%$ and $40 \%$ of cell viability for the groups treated with DOX loaded MONs and DOX loaded Her2 affibody targeted MONs, respectively. Xu et al. developed hyaluronic acid functionalized PMOs to perform the targeted chemotherapy of liver cancer [100]. The hyaluronic acid is the primary binding molecule of CD44, a glycoprotein overexpressed in various tumors such as breast, liver, and lung cancer. The PMOs were produced through the co-condensation of [4-(4,4,5,5-tetramethyl-1,3,2-dioxaborolan-2-yl)-N-(3-(trimethoxysilyl) propyl)benzamide] and 3-aminopropyltrimethoxysilane in the presence of PEI. Then the PMOs surface was modified with hyaluronic acid by exploring the electrostatic interactions. These authors observed that the hyaluronic acid targeted PMOs presented a 5-fold increased uptake by

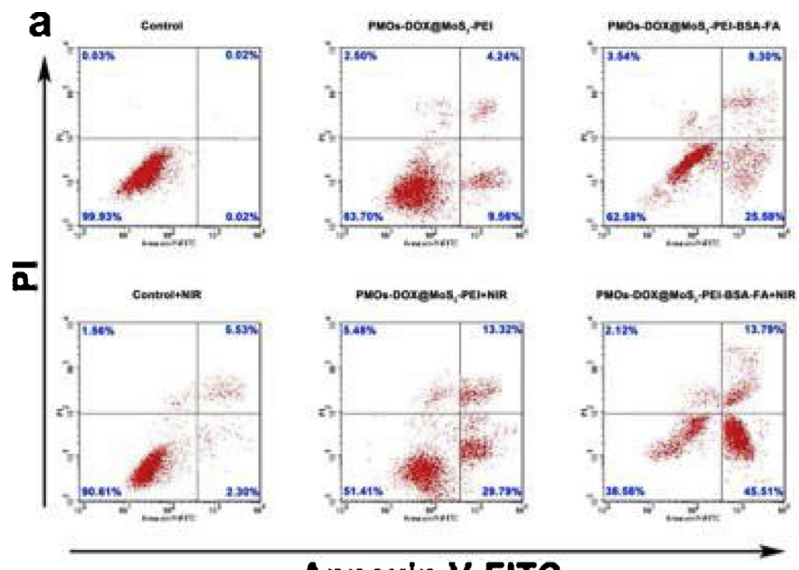

Annexin V-FITC
HepG2 cancer cells (overexpressing CD44 receptors) than in normal NIH 3T3 cells (low CD44 expression), whereas only a 1.7-fold increase was detected for the nontargeted nanoparticles. Such preferential uptake resulted in an increased cytotoxicity of hyaluronic acid targeted PMOs towards HepG2 cancer cells, half maximal inhibitory concentration $\left(\mathrm{IC}_{50}\right.$ ) of $0.49 \mu \mathrm{M}$ and $2.1 \mu \mathrm{M}$ for HepG2 and NIH 3T3 cells, respectively. Further, they also reported that the $\mathrm{IC}_{50}$ on HepG2 cancer cells for the nontargeted PMOs was 4 times higher $(2 \mu \mathrm{M})$ than that of the targeted PMOs. Similarly, Wu and colleagues produced a folic acid targeted MON functionalized with $\mathrm{MoS}_{2}$ nanosheets, PEI, and albumin for the breast cancer chemo-photothermal therapy [58]. These authors selected folic acid since it is a ligand for the folate receptor: a glycosylphosphatidyinositol-linked protein that captures and transports its ligands from the extracellular media to the cell cytoplasm; and that is overexpressed in the majority of cancers (e.g. ovarian, cervical, breast, and lung). For that purpose, the MONs were synthesized through the co-condensation of TEOS and TESPTS, followed by the adsorption of $\mathrm{MoS}_{2} / \mathrm{PEI} /$ albumin/folic acid complexes. The authors demonstrated that the MCF-7 cells (folate receptor overexpressing cells) incubated with the targeted nanoparticles present a stronger DOX fluorescence than the ones without the targeting moiety. Moreover, they only detected a weak DOX fluorescence in the hepatoma 7402 cells (folate receptor negative). Moreover, $\mathrm{Wu}$ et al. also recorded an increased cellular apoptosis after the MCF-7 cells incubation with targeted MONs for $12 \mathrm{~h}, 13.8 \%$ and $33.9 \%$ apoptotic cells for nontargeted and targeted MONs, respectively (Fig. 7).

\section{MONs biocompatibility}

The nanoparticles biocompatibility is one of the primordial concerns when biomedical applications are envisioned. Therefore, extensive in vitro and in vivo toxicological evaluations are essential for validating the nanomedicines applicability. In fact, one of the main objectives of the organosilica nanoparticles is to enhance the biodegradability and biosafety of silica-based nanomedicines (Fig. 8).

Moghaddam and colleagues developed redox-responsive MONs for delivering anticancer chemotherapeutics [37]. The authors produced two different redox-responsive MONs by promoting the co-condensation of TEOS and BTDS (disulfide-based MONs) or TEOS and BTES (tetrasulfide-based MONs) under a basic environment in the presence of CTAB. The authors reported that the resulting disulfide-based and tetradisulfide-based MONs presented a similar size $(\sim 110 \mathrm{~nm})$, surface

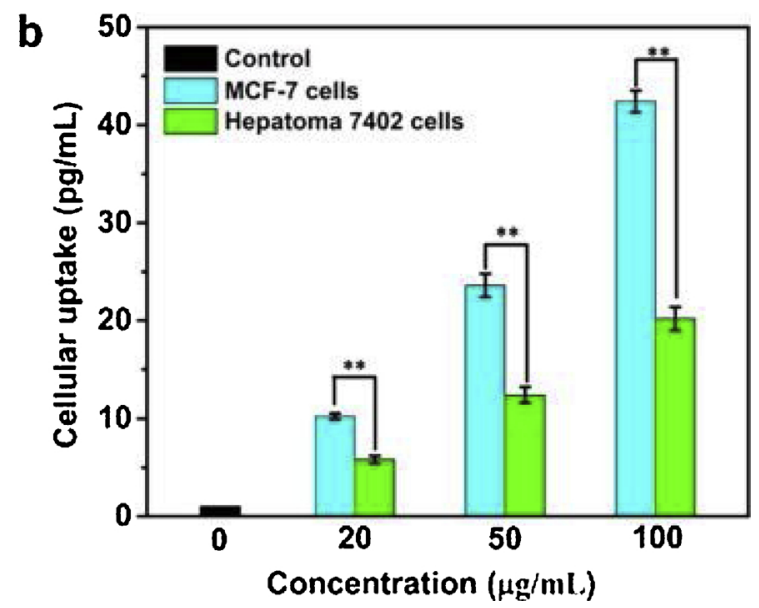

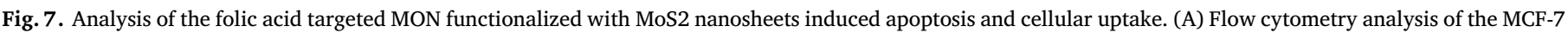

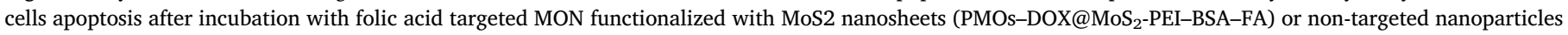

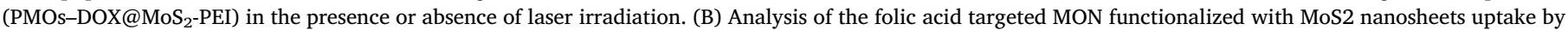

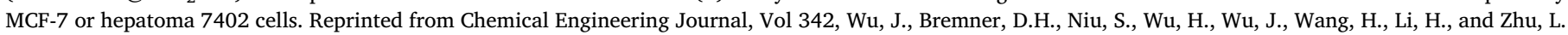

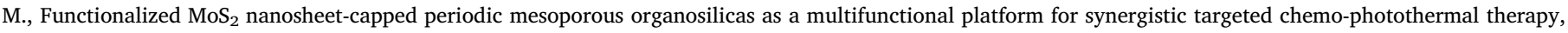
Pages No.90-102, Copyright (2017), with permission from Elsevier. 


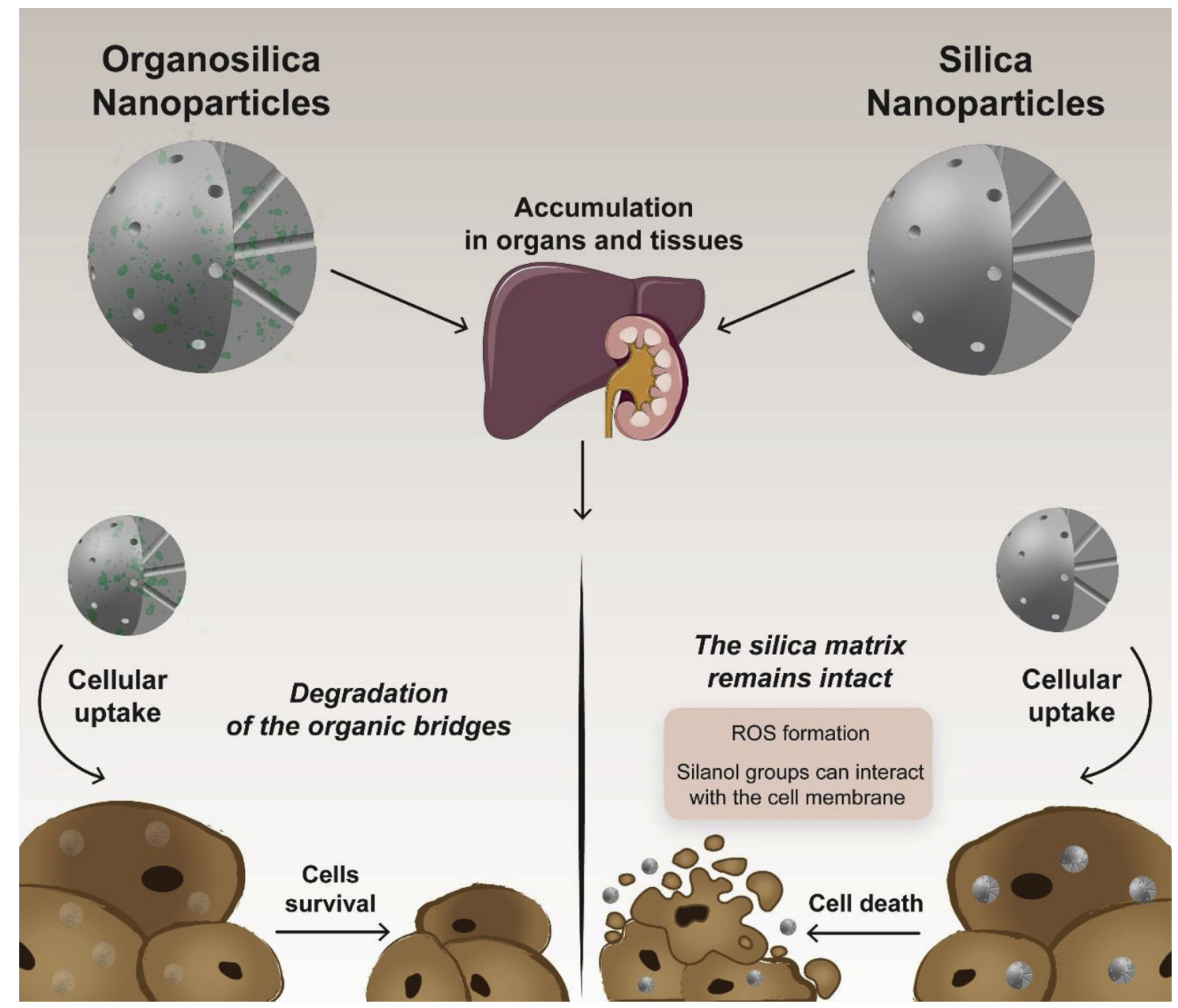

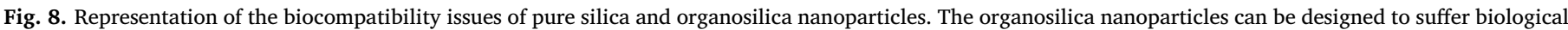

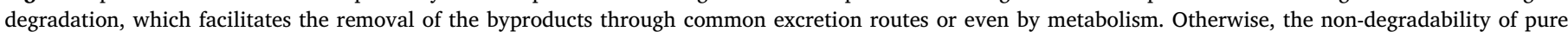

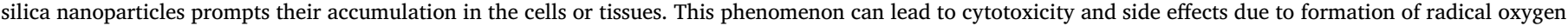
species or even by provoking the cell lysis.

charge (-32 and $-27 \mathrm{mV}$ ) and pore diameters (2 and $2.7 \mathrm{~nm})$. Nevertheless, the in vitro degradation studies performed in the GSH presence revealed that the disulfide-based MONs have a higher degradation rate, reaching the $14 \%$ in 15 days. Further, the authors also observed that the disulfide-based nanoparticles presented a superior biocompatibility with RAW 264.7 macrophages, $\mathrm{IC}_{50}$ of $705 \mu \mathrm{g} / \mathrm{mL}$ and $233 \mu \mathrm{g} / \mathrm{mL}$ for disulfide-based MONs and tetrasulfide-based MONs, respectively (Fig. 9).

Similarly, Yue et al. reported the production of MONs with organic bridges containing disulfide bonds through the co-condensation of TEOS and bis[3-(triethoxysily)propyl]tetrasulfide [60]. These authors observed that the MONs could be completely degraded after $72 \mathrm{~h}$ in the presence of GSH, contrasting to stable mesoporous silica counterparts. Further, the MONs presented a high biocompatibility (at maximum concentration of $150 \mu \mathrm{g} / \mathrm{mL}$ ) with more than $85 \%$ of the cells (HepG2 or HL-7702) remaining viable, even when the particles were incubated with the MONs degradation products. Zhang and coworkers also reported the production of hollow MONs composed by organic bridges containing disulfide bonds for cancer drug delivery [24]. The TEM images acquired by these researchers revealed that the MONs incubation in the presence of GSH induce the fast degradation of the nanoparticles in one week. Further, the authors observed that the MONs administration in healthy Balb/c mice at the doses of 5, 10, and $20 \mathrm{mg}$ / $\mathrm{kg}$ did not induce any negative effects on animal's weight. Moreover, the liver and kidneys function indicators (e.g. alanine aminotransferase, aspartate aminotransferase, blood urea nitrogen, and creatinine), as well as the blood routine indexes (e.g. white blood count and red blood count), remained in the normal range. Such data conjugated with the absence of damages on the histological analysis of the major organs supports the biosafety of biodegradable MONs.

\section{Conclusion and future perspectives}

The organosilica nanoparticles conjugate the MSNs' stability (hydrothermal and hydrolytic) and ordered porous structure with the organic materials improved colloidal stability, biodegradability, biocompatibility, and stimuli-responsiveness. In the present review, the organosilica nanoparticles' application in cancer nanomedicine, stimuli-responsive drug delivery, tumor-specific accumulation, and biocompatibility, was highlighted. In fact, the inclusion of organic bridges on the mesoporous silica matrix offers the possibility to tune the nanoparticles biodegradation, which can be exploited to control the space and time of the drug delivery to cancer cells. Moreover, the almost unlimited organosilica alternatives allow the development of PMOs or MONs with bioimaging (e.g. magnetic resonance, fluorescent, and ultrasounds) and therapeutic (e.g. photothermal, controlled drug delivery, and gene therapy) functionalities. Further, as previously summarized, the organosilica nanoparticles also allow the surface modification with passivation and targeting agents that increase the blood circulation time and specificity towards the tumor tissue. 


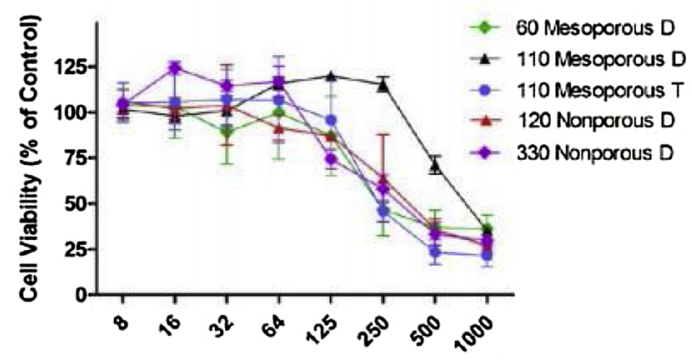

Concentration of Nanoparticles $\left(\mu \mathrm{g} \mathrm{mr}^{-1}\right)$
B

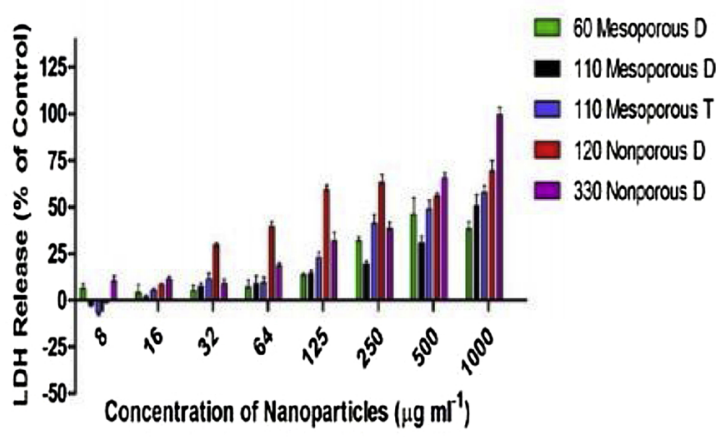

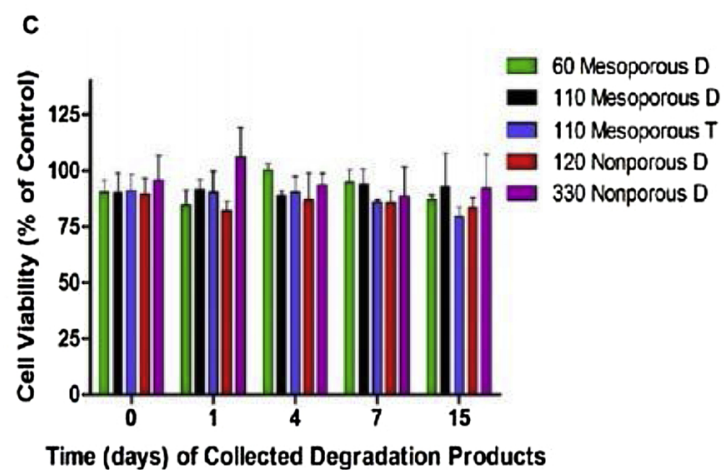

Fig. 9. Analysis of the RAW 264.7 macrophages viability after incubation with different redox-responsive MONs. (A) CCK-8 and (B) LDH assays after the cells incubation for $24 \mathrm{~h}$. (C) Cytotoxicity evaluation of the cells incubated with the particles degradation products. Reprinted with permission from ACS Appl. Mater. Interfaces, Vol 9, Moghaddam, S.P.H., Saikia, J., Yazdimamaghani, M., and Ghandehari, H., Redox-Responsive Polysulfide-Based Biodegradable Organosilica Nanoparticles for Delivery of Bioactive Agents, Pages No. 211333-21146, Copyright (2017) American Chemical Society.

However, the synthesis scalability and the development of straightforward production methods are still one of the main challenges in this field, particularly for PMOs. Further, despite the promising initial reports on the organosilica biosafety, the current results are still too preliminary and more extensive biodistribution, excretion, hemo-/histocompatibility, and biodegradation studies must be performed to validate these nanomedicines.

Nevertheless, the high pre-clinical performance demonstrated by the organosilica nanoparticles in biomedical applications encourages further efforts towards the development of more precise and personalized nanomedicines for cancer therapy.

\section{Declaration of Competing Interest}

None.

\section{Acknowledgments}

This work was supported by FEDER funds through the POCI COMPETE 2020 - Operational Programme Competitiveness and Internationalisation in Axis I - Strengthening research, technological development and innovation (Project POCI-01-0145-FEDER-007491) and National Funds by FCT - Foundation for Science and Technology (Project UID/Multi/00709/2013). The funding from CENTRO-01-0145FEDER-028989 and POCI-01-0145-FEDER-031462 are also acknowledged. André F. Moreira and Carolina F. Rodrigues acknowledge for their individual Ph.D. fellowships from FCT (SFRH/BD/109482/2015 and SFRH/BD/144680/2019, respectively). The funders had no role in the decision to publish or in the preparation of the manuscript.

\section{Appendix A. Supplementary data}

Supplementary material related to this article can be found, in the online version, at doi:https://doi.org/10.1016/j.phrs.2020.104742.

\section{References}

[1] Z. Teng, J. Zhang, W. Li, Y. Zheng, X. Su, Y. Tang, M. Dang, Y. Tian, L. Yuwen, L. Weng, G. Lu, L. Wang, Facile Synthesis of Yolk-Shell-Structured TripleHybridized Periodic Mesoporous Organosilica Nanoparticles for Biomedicine, Small 12 (2016) 3550-3558.

[2] A.F. Moreira, D.R. Dias, I.J. Correia, Stimuli-responsive mesoporous silica nanoparticles for cancer therapy: a review, Microporous Mesoporous Mater. 236 (2016) 141-157.

[3] F. Tang, L. Li, D. Chen, Mesoporous silica nanoparticles: synthesis, biocompatibility and drug delivery, Adv. Mater. 24 (2012) 1504-1534.

[4] N. Chen, W. Yang, Y. Bao, H. Xu, S. Qin, Y. Tu, BSA capped Au nanoparticle as an efficient sensitizer for glioblastoma tumor radiation therapy, RSC Adv. 5 (2015) 40514-40520.

[5] A. Madaan, P. Singh, A. Awasthi, R. Verma, A.T. Singh, M. Jaggi, S.K. Mishra, S. Kulkarni, H. Kulkarni, Efficiency and mechanism of intracellular paclitaxel delivery by novel nanopolymer-based tumor-targeted delivery system, NanoxelTM, Clin. Transl. Oncol. 15 (2013) 26-32.

[6] C.S.S.R. Kumar, F. Mohammad, Magnetic nanomaterials for hyperthermia-based therapy and controlled drug delivery, Adv. Drug Deliv. Rev. 63 (2011) 789-808.

[7] Z. Peng, E.H. Miyanji, Y. Zhou, J. Pardo, S.D. Hettiarachchi, S. Li, P.L. Blackwelder, I. Skromne, R.M. Leblanc, Carbon dots: promising biomaterials for bone-specific imaging and drug delivery, Nanoscale 9 (2017) 17533-17543.

[8] S.-H. Hu, R.-H. Fang, Y.-W. Chen, B.-J. Liao, I.W. Chen, S.-Y. Chen, Photoresponsive protein-Graphene-Protein hybrid capsules with dual targeted heat-triggered drug delivery approach for enhanced tumor therapy, Adv. Funct. Mater. 24 (2014) 4144-4155.

[9] S. Jafari, H. Derakhshankhah, L. Alaei, A. Fattahi, B.S. Varnamkhasti, A.A. Saboury, Mesoporous silica nanoparticles for therapeutic/diagnostic applications, Biomed. Pharmacother. 109 (2019) 1100-1111.

[10] Y. Zhou, G. Quan, Q. Wu, X. Zhang, B. Niu, B. Wu, Y. Huang, X. Pan, C. Wu, Mesoporous silica nanoparticles for drug and gene delivery, Acta Pharm. Sin. B 8 (2018) 165-177.

[11] D. Shao, M. Li, Z. Wang, X. Zheng, Y.-H. Lao, Z. Chang, F. Zhang, M. Lu, J. Yue, H. Hu, H. Yan, L. Chen, W.-f. Dong, K.W. Leong, Bioinspired diselenide-bridged mesoporous silica nanoparticles for dual-responsive protein delivery, Adv. Mater. 30 (2018) 1801198.

[12] R. Rahmatolahzadeh, M. Hamadanian, L. Ma'mani, A. Shafiee, Aspartic acid functionalized PEGylated MSN@GO hybrid as an effective and sustainable nanosystem for in-vitro drug delivery, Adv. Med. Sci. 63 (2018) 257-264.

[13] F. Wang, L. Zhang, X. Bai, X. Cao, X. Jiao, Y. Huang, Y. Li, Y. Qin, Y. Wen, Stimuliresponsive Nanocarrier for Co-delivery of MiR-31 and doxorubicin to suppress high MtEF4 Cancer, ACS Appl. Mater. Interfaces 10 (2018) 22767-22775. 
[14] F. Carniato, D. Alberti, A. Lapadula, J. Martinelli, C. Isidoro, S. Geninatti Crich, L. Tei, Multifunctional Gd-based mesoporous silica nanotheranostic for anticancer drug delivery, J. Mater. Chem. B 7 (2019) 3143-3152.

[15] H. Mekaru, J. Lu, F. Tamanoi, Development of mesoporous silica-based nanoparticles with controlled release capability for cancer therapy, Adv. Drug Deliv. Rev. 95 (2015) 40-49.

[16] A. Watermann, J. Brieger, Mesoporous silica nanoparticles as drug delivery vehicles in Cancer, Nanomaterials 7 (2017) 189.

[17] P. Huang, Y. Chen, H. Lin, L. Yu, L. Zhang, L. Wang, Y. Zhu, J. Shi, Molecularly organic/inorganic hybrid hollow mesoporous organosilica nanocapsules with tumor-specific biodegradability and enhanced chemotherapeutic functionality, Biomaterials 125 (2017) 23-37.

[18] J.G. Croissant, Y. Fatieiev, N.M. Khashab, Degradability and clearance of Silicon, organosilica, Silsesquioxane, silica mixed oxide, and mesoporous silica nanoparticles, Adv. Mater. 29 (2017) 1604634.

[19] X. Du, W. Li, B. Shi, L. Su, X. Li, H. Huang, Y. Wen, X. Zhang, Facile synthesis of mesoporous organosilica nanobowls with bridged silsesquioxane framework by one-pot growth and dissolution mechanism, J. Colloid Interface Sci. 528 (2018) 379-388.

[20] S.S. Park, M. Santha Moorthy, C.-S. Ha, Periodic mesoporous organosilicas for advanced applications, NPG Asia Mater. 6 (2014) e96.

[21] M. Wu, Q. Meng, Y. Chen, Y. Du, L. Zhang, Y. Li, L. Zhang, J. Shi, Large-pore ultrasmall mesoporous organosilica nanoparticles: micelle/precursor co-templating assembly and nuclear-targeted gene delivery, Adv. Mater. 27 (2015) 215-222.

[22] K. Burglova, A. Noureddine, J. Hodacova, G. Toquer, X. Cattoen, M. Wong Chi Man, A general method for preparing bridged organosilanes with pendant functional groups and functional mesoporous organosilicas, Chemistry 20 (2014) 10371-10382.

[23] J. Croissant, X. Cattoen, M.W. Man, A. Gallud, L. Raehm, P. Trens, M. Maynadier, J.O. Durand, Biodegradable ethylene-bis(propyl)disulfide-based periodic mesoporous organosilica nanorods and nanospheres for efficient in-vitro drug delivery, Adv. Mater. 26 (2014) 6174-6180.

[24] L. Zhang, L. Wang, H. Yao, F. Xu, Y. Chen, Biodegradable and biocompatible monodispersed hollow mesoporous organosilica with large pores for delivering biomacromolecules, J. Mater. Chem. B 5 (2017) 8013-8025.

[25] J. Wu, G.R. Williams, S. Niu, F. Gao, R. Tang, L.M. Zhu, A multifunctional biodegradable nanocomposite for Cancer theranostics, Adv. Sci. 6 (2019) 1802001.

[26] Y. Li, W. Guo, X. Su, L. Ou-Yang, M. Dang, J. Tao, G. Lu, Z. Teng, Small size mesoporous organosilica nanorods with different aspect ratios: synthesis and cellular uptake, J. Colloid Interface Sci. 512 (2018) 134-140.

[27] L. Yu, Y. Chen, H. Lin, W. Du, H. Chen, J. Shi, Ultrasmall mesoporous organosilica nanoparticles: morphology modulations and redox-responsive biodegradability for tumor-specific drug delivery, Biomaterials 161 (2018) 292-305.

[28] H. Omar, J.G. Croissant, K. Alamoudi, S. Alsaiari, I. Alradwan, M.A. Majrashi, D.H. Anjum, P. Martins, R. Laamarti, J. Eppinger, B. Moosa, A. Almalik, N.M. Khashab, Biodegradable magnetic Silica@Iron oxide nanovectors with ultralarge mesopores for high protein loading, magnetothermal release, and delivery, J. Control. Release 259 (2017) 187-194.

[29] W. Liu, N. Ma, S. Li, X. Zhang, W. Huo, J. Xu, X. Meng, J. Yang, A one-step method for pore expansion and enlargement of hollow cavity of hollow periodic mesoporous organosilica spheres, J. Mater. Sci. 52 (2016) 2868-2878.

[30] J.G. Croissant, Y. Fatieiev, A. Almalik, N.M. Khashab, Mesoporous silica and organosilica nanoparticles: physical chemistry, biosafety, delivery strategies, and biomedical applications, Adv. Healthc. Mater. 7 (2018) 1700831.

[31] W. Fan, N. Lu, Z. Shen, W. Tang, B. Shen, Z. Cui, L. Shan, Z. Yang, Z. Wang, O. Jacobson, Z. Zhou, Y. Liu, P. Hu, W. Yang, J. Song, Y. Zhang, L. Zhang, N.M. Khashab, M.A. Aronova, G. Lu, X. Chen, Generic synthesis of small-sized hollow mesoporous organosilica nanoparticles for oxygen-independent X-ray-activated synergistic therapy, Nat. Commun. 10 (2019) 1241.

[32] M.S. Moorthy, H.-J. Song, J.-H. Bae, S.-H. Kim, C.-S. Ha, Red fluorescent hybrid mesoporous organosilicas for simultaneous cell imaging and anticancer drug delivery, RSC Adv. 4 (2014) 43342-43345.

[33] Q. Ni, Z. Teng, M. Dang, Y. Tian, Y. Zhang, P. Huang, X. Su, N. Lu, Z. Yang, W. Tian, S. Wang, W. Liu, Y. Tang, G. Lu, L. Zhang, Gold nanorod embedded largepore mesoporous organosilica nanospheres for gene and photothermal cooperative therapy of triple negative breast cancer, Nanoscale 9 (2017) 1466-1474.

[34] Y. Yang, Y. Niu, J. Zhang, A.K. Meka, H. Zhang, C. Xu, C.X. Lin, M. Yu, C. Yu, Biphasic Synthesis of Large-Pore and Well-Dispersed Benzene Bridged Mesoporous Organosilica Nanoparticles for Intracellular Protein Delivery, Small 11 (2015) 2743-2749.

[35] M. Wu, Y. Chen, L. Zhang, X. Li, X. Cai, Y. Du, L. Zhang, J. Shi, A salt-assisted acid etching strategy for hollow mesoporous silica/organosilica for $\mathrm{pH}$-responsive drug and gene co-delivery, J. Mater. Chem. B 3 (2015) 766-775.

[36] N. Lu, P. Huang, W. Fan, Z. Wang, Y. Liu, S. Wang, G. Zhang, J. Hu, W. Liu, G. Niu, R.D. Leapman, G. Lu, X. Chen, Tri-stimuli-responsive biodegradable theranostics for mild hyperthermia enhanced chemotherapy, Biomaterials 126 (2017) 39-48.

[37] S.P. Hadipour Moghaddam, J. Saikia, M. Yazdimamaghani, H. Ghandehari, Redoxresponsive polysulfide-based biodegradable organosilica nanoparticles for delivery of bioactive agents, ACS Appl. Mater. Interfaces 9 (2017) 21133-21146.

[38] C. Mauriello Jimenez, D. Aggad, J.G. Croissant, K. Tresfield, D. Laurencin, D. Berthomieu, N. Cubedo, M. Rossel, S. Alsaiari, D.H. Anjum, R. Sougrat, M.A. Roldan-Gutierrez, S. Richeter, E. Oliviero, L. Raehm, C. Charnay, X. Cattoën, S. Clément, M. Wong Chi Man, M. Maynadier, V. Chaleix, V. Sol, M. Garcia, M. Gary-Bobo, N.M. Khashab, N. Bettache, J.-O. Durand, Porous porphyrin-based organosilica nanoparticles for NIR two-photon photodynamic therapy and gene delivery in zebrafish, Adv. Funct. Mater. 28 (2018) 1800235.

[39] Z.L. Yang, W. Tian, Q. Wang, Y. Zhao, Y.L. Zhang, Y. Tian, Y.X. Tang, S.J. Wang, Y. Liu, Q.Q. Ni, G.M. Lu, Z.G. Teng, L.J. Zhang, Oxygen-evolving mesoporous organosilica coated prussian blue nanoplatform for highly efficient photodynamic therapy of tumors, Adv. Sci. 5 (2018) 1700847.

[40] N. Mizoshita, Y. Goto, M.P. Kapoor, T. Shimada, T. Tani, S. Inagaki, Fluorescence emission from 2,6-Naphthylene-Bridged mesoporous organosilicas with an amorphous or crystal-like framework, Chem. Eur. J. 15 (2009) 219-226.

[41] M. Nakamura, K. Hayashi, M. Nakano, T. Kanadani, K. Miyamoto, T. Kori, K. Horikawa, Identification of polyethylene glycol-resistant macrophages on stealth imaging in vitro using fluorescent organosilica nanoparticles, ACS Nano 9 (2015) 1058-1071.

[42] M. Wang, W. Liu, Y. Zhang, M. Dang, Y. Zhang, J. Tao, K. Chen, X. Peng, Z. Teng, Intercellular adhesion molecule 1 antibody-mediated mesoporous drug delivery system for targeted treatment of triple-negative breast cancer, J. Colloid Interface Sci. 538 (2019) 630-637.

[43] B. Julián-López, C. Boissière, C. Chanéac, D. Grosso, S. Vasseur, S. Miraux, E. Duguet, C. Sanchez, Mesoporous maghemite-organosilica microspheres: a promising route towards multifunctional platforms for smart diagnosis and therapy, J. Mater. Chem. 17 (2007) 1563-1569.

[44] S. Datz, H. Engelke, Cv Schirnding, L. Nguyen, T. Bein, Lipid bilayer-coated curcumin-based mesoporous organosilica nanoparticles for cellular delivery, Microporous Mesoporous Mater. 225 (2016) 371-377.

[45] B. Yang, Y. Chen, J. Shi, Mesoporous silica/organosilica nanoparticles: synthesis, biological effect and biomedical application, Mater. Sci. Eng. R Rep. 137 (2019) 66-105.

[46] Z. Teng, W. Li, Y. Tang, A. Elzatahry, G. Lu, D. Zhao, Mesoporous organosilica hollow nanoparticles: synthesis and applications, Adv. Mater. (2018) 1707612.

[47] L.-L. Hu, D.-D. Zhang, Y. Zhang, Y. Shu, X.-W. Chen, J.-H. Wang, Glutathione functionalized mesoporous organosilica conjugate for drug delivery, RSC Adv. 6 (2016) 56287-56293.

[48] J. Zhang, X. Wang, J. Wen, X. Su, L. Weng, C. Wang, Y. Tian, Y. Zhang, J. Tao, P. Xu, G. Lu, Z. Teng, L. Wang, Size effect of mesoporous organosilica nanoparticles on tumor penetration and accumulation, Biomater. Sci. 7 (2019) 4790-4799.

[49] Q. Wei, Z.-X. Zhong, Z.-R. Nie, J.-L. Li, F. Wang, Q.-Y. Li, Catalytically active gold nanoparticles confined in periodic mesoporous organosilica (PMOs) by a modified external passivation route, Microporous Mesoporous Mater. 117 (2009) 98-103.

[50] J. Tao, M. Dang, X. Su, Q. Hao, J. Zhang, X. Ma, G. Lu, Y. Zhang, Y. Tian, L. Weng, Z. Teng, L. Wang, Facile synthesis of yolk-shell structured monodisperse mesoporous organosilica nanoparticles by a mild alkalescent etching approach, J. Colloid Interface Sci. 527 (2018) 33-39.

[51] L. Shen, S. Pan, D. Niu, J. He, X. Jia, J. Hao, J. Gu, W. Zhao, P. Li, Y. Li, Facile synthesis of organosilica-capped mesoporous silica nanocarriers with selective redox-triggered drug release properties for safe tumor chemotherapy, Biomater. Sci. 7 (2019) 1825-1832.

[52] Y. Chen, Q. Meng, M. Wu, S. Wang, P. Xu, H. Chen, Y. Li, L. Zhang, L. Wang, J. Shi, Hollow mesoporous organosilica nanoparticles: a generic intelligent frameworkhybridization approach for biomedicine, J. Am. Chem. Soc. 136 (2014) 1632611634.

[53] B. Guan, Y. Cui, Z. Ren, Z.-a. Qiao, L. Wang, Y. Liu, Q. Huo, Highly ordered periodic mesoporous organosilica nanoparticles with controllable pore structures, Nanoscale 4 (2012) 6588-6596.

[54] J. Croissant, X. Cattoën, M.W.C. Man, A. Gallud, L. Raehm, P. Trens, M. Maynadier, J.-O. Durand, Biodegradable ethylene-bis(Propyl)Disulfide-Based periodic mesoporous organosilica nanorods and nanospheres for efficient in-vitro drug delivery, Adv. Mater. 26 (2014) 6174-6180.

[55] K. Chen, J. Tao, W. Shi, X. Su, X. Cao, X. Peng, L. Bao, Z. Feng, Y. Zhao, X. Mai, L. Wang, G. Lu, Z. Teng, Soft mesoporous organosilica nanorods with gold plasmonic core for significantly enhanced cellular uptake, J. Colloid Interface Sci. 550 (2019) 81-89.

[56] L. Chen, J. Zhang, X. Zhou, S. Yang, Q. Zhang, W. Wang, Z. You, C. Peng, C. He, Merging metal organic framework with hollow organosilica nanoparticles as a versatile nanoplatform for cancer theranostics, Acta Biomater. 86 (2019) 406-415.

[57] X. Qian, W. Wang, W. Kong, Y. Chen, Organic-Inorganic Hybrid Hollow Mesoporous Organosilica Nanoparticles for Efficient Ultrasound-Based Imaging and Controlled Drug Release, Nanomaterials 2014 (2014) 1-8.

[58] J. Wu, D.H. Bremner, S. Niu, H. Wu, J. Wu, H. Wang, H. Li, L.-M. Zhu, Functionalized MoS2 nanosheet-capped periodic mesoporous organosilicas as a multifunctional platform for synergistic targeted chemo-photothermal therapy, Chem. Eng. J. 342 (2018) 90-102.

[59] Z. Teng, C. Wang, Y. Tang, W. Li, L. Bao, X. Zhang, X. Su, F. Zhang, J. Zhang, S. Wang, D. Zhao, G. Lu, Deformable hollow periodic mesoporous organosilica nanocapsules for significantly improved cellular uptake, J. Am. Chem. Soc. 140 (2018) 1385-1393.

[60] J. Yue, S.Z. Luo, M.M. Lu, D. Shao, Z. Wang, W.F. Dong, A comparison of mesoporous silica nanoparticles and mesoporous organosilica nanoparticles as drug vehicles for cancer therapy, Chem. Biol. Drug Des. 92 (2018) 1435-1444.

[61] Y. Sun, M. Chen, L. Wu, Controllable synthesis of hollow periodic mesoporous organosilica spheres with radial mesochannels and their degradable behavior, J. Mater. Chem. A 6 (2018) 12323-12333.

[62] B. Karimi, F.K. Esfahani, Gold nanoparticles supported on the periodic mesoporous organosilicas as efficient and reusable catalyst for room temperature aerobic oxidation of alcohols, Adv. Synth. Catal. 354 (2012) 1319-1326.

[63] D. Aggad, C.M. Jimenez, S. Dib, J.G. Croissant, L. Lichon, D. Laurencin, S. Richeter, M. Maynadier, S.K. Alsaiari, M. Boufatit, L. Raehm, M. Garcia, 
N.M. Khashab, M. Gary-Bobo, J.-O. Durand, Gemcitabine delivery and photodynamic therapy in Cancer cells via porphyrin-ethylene-Based periodic mesoporous organosilica nanoparticles, Chemistry Nanomaterials energy, Biology and more 4 (2018) 46-51.

[64] H. Takeda, M. Ohashi, T. Tani, O. Ishitani, S. Inagaki, Enhanced photocatalysis of rhenium(I) complex by light-harvesting periodic mesoporous organosilica, Inorg. Chem. 49 (2010) 4554-4559.

[65] J.G. Croissant, Y. Fatieiev, H. Omar, D.H. Anjum, A. Gurinov, J. Lu, F. Tamanoi, J.I. Zink, N.M. Khashab, Periodic mesoporous organosilica nanoparticles with controlled morphologies and high Drug/Dye loadings for multicargo delivery in Cancer cells, Chemistry 22 (2016) 9607-9615.

[66] J.G. Croissant, Y. Fatieiev, K. Julfakyan, J. Lu, A.-H. Emwas, D.H. Anjum, H. Omar, F. Tamanoi, J.I. Zink, N.M. Khashab, Biodegradable oxamide-phenylene-Based mesoporous organosilica nanoparticles with unprecedented drug payloads for delivery in cells, Chem. Eur. J. 22 (2016) 14806-14811.

[67] K.M. Rao, S. Parambadath, A. Kumar, C.-S. Ha, S.S. Han, Tunable intracellular degradable periodic mesoporous organosilica hybrid nanoparticles for doxorubicin drug delivery in Cancer cells, ACS Biomater. Sci. Eng. 4 (2017) 175-183.

[68] X. Cheng, D. Li, A. Lin, J. Xu, L. Wu, H. Gu, Z. Huang, J. Liu, Y. Zhang, X. Yin, Fabrication of multifunctional triple-responsive platform based on CuS-capped periodic mesoporous organosilica nanoparticles for chemo-photothermal therapy, Int. J. Nanomedicine 13 (2018) 3661-3677.

[69] C.F. Rodrigues, T.A. Jacinto, A.F. Moreira, E.C. Costa, S.P. Miguel, I.J. Correia, Functionalization of AuMSS nanorods towards more effective cancer therapies, Nano Res. 12 (2019) 719-732.

[70] A.F. Moreira, C.F. Rodrigues, C.A. Reis, E.C. Costa, P. Ferreira, I.J. Correia, Development of poly-2-ethyl-2-oxazoline coated gold-core silica shell nanorods for cancer chemo-photothermal therapy, Nanomedicine 13 (2018) 2611-2627.

[71] J. Du, L.A. Lane, S. Nie, Stimuli-responsive nanoparticles for targeting the tumor microenvironment, J. Control. Release 219 (2015) 205-214.

[72] Y. Shen, H. Tang, M. Radosz, E. Van Kirk, W.J. Murdoch, pH-responsive nanoparticles for cancer drug delivery, in: K.K. Jain (Ed.), Drug Delivery Systems, Humana Press, Totowa, NJ, 2008, pp. 183-216.

[73] A.F. Moreira, V.M. Gaspar, E.C. Costa, D. de Melo-Diogo, P. Machado, C.M. Paquete, I.J. Correia, Preparation of end-capped pH-sensitive mesoporous silica nanocarriers for on-demand drug delivery, Eur. J. Pharm. Biopharm. 88 (2014) 1012-1025.

[74] A.F. Moreira, D.R. Dias, E.C. Costa, I.J. Correia, Thermo- and pH-responsive nanoin-micro particles for combinatorial drug delivery to cancer cells, Eur. J. Pharm. Sci. 104 (2017) 42-51.

[75] F. Danhier, O. Feron, V. Préat, To exploit the tumor microenvironment: passive and active tumor targeting of nanocarriers for anti-cancer drug delivery, $\mathrm{J}$. Control. Release 148 (2010) 135-146.

[76] J. Zhao, M.H. Stenzel, Entry of nanoparticles into cells: the importance of nanoparticle properties, Polym. Chem. 9 (2018) 259-272.

[77] J. Mosquera, I. García, L.M. Liz-Marzán, Cellular uptake of nanoparticles versus small molecules: a matter of size, Acc. Chem. Res. 51 (2018) 2305-2313.

[78] L. Liu, C. Kong, M. Huo, C. Liu, L. Peng, T. Zhao, Y. Wei, F. Qian, J. Yuan, Schiff base interaction tuned mesoporous organosilica nanoplatforms with $\mathrm{pH}$-responsive degradability for efficient anti-cancer drug delivery in vivo, Chem. Commun. 54 (2018) 9190-9193.

[79] M.S. Moorthy, J.-H. Bae, M.-J. Kim, S.-H. Kim, C.-S. Ha, Design of a novel mesoporous organosilica hybrid microcarrier: a $\mathrm{pH}$ stimuli-responsive dual-drugDelivery vehicle for intracellular delivery of anticancer agents, Part. Part. Syst. Charact. 30 (2013) 1044-1055.

[80] S. Parambadath, A. Mathew, M. Jenisha Barnabas, C.-S. Ha, A pH-responsive drug delivery system based on ethylenediamine bridged periodic mesoporous organosilica, Microporous Mesoporous Mater. 215 (2015) 67-75.

[81] D.J. Phillips, M.I. Gibson, Redox-sensitive materials for drug delivery: targeting the correct intracellular environment, tuning release rates, and appropriate predictive systems, Antioxid. Redox Signal. 21 (2013) 786-803.

[82] L. Brülisauer, M.A. Gauthier, J.-C. Leroux, Disulfide-containing parenteral delivery systems and their redox-biological fate, J. Control. Release 195 (2014) 147-154.

[83] H. Mekaru, A. Yoshigoe, M. Nakamura, T. Doura, F. Tamanoi, Biodegradability of disulfide-organosilica nanoparticles evaluated by Soft x-ray photoelectron spectroscopy: cancer therapy implications, Acs Appl. Nano Mater. 2 (2018) 479-488.

[84] H.J. Forman, H. Zhang, A. Rinna, Glutathione: overview of its protective roles, measurement, and biosynthesis, Mol. Aspects Med. 30 (2009) 1-12.

[85] J. Yue, S.-z. Luo, M.-m. Lu, D. Shao, Z. Wang, W.-f. Dong, A comparison of mesoporous silica nanoparticles and mesoporous organosilica nanoparticles as drug vehicles for cancer therapy, Chem. Biol. Drug Des. 92 (2018) 1435-1444.

[86] T. Shao, J. Wen, Q. Zhang, Y. Zhou, L. Liu, L. Yuwen, Y. Tian, Y. Zhang, W. Tian, Y. Su, Z. Teng, G. Lu, J. Xu, NIR photoresponsive drug delivery and synergistic chemo-photothermal therapy by monodispersed-MoS2-nanosheets wrapped periodic mesoporous organosilicas, J. Mater. Chem. B 4 (2016) 7708-7717.

[87] H. Omar, B. Moosa, K. Alamoudi, D.H. Anjum, A.H. Emwas, O. El Tall, B. Vu, F. Tamanoi, A. AlMalik, N.M. Khashab, Impact of pore-walls ligand assembly on the biodegradation of mesoporous organosilica nanoparticles for controlled drug delivery, ACS Omega 3 (2018) 5195-5201.

[88] S.-Z. Ren, D. Zhu, X.-H. Zhu, B. Wang, Y.-S. Yang, W.-X. Sun, X.-M. Wang, P.-C. Lv,
Z.-C. Wang, H.-L. Zhu, Nanoscale metal-Organic-Frameworks coated by biodegradable organosilica for $\mathrm{pH}$ and redox dual responsive drug release and highperformance anticancer therapy, ACS Appl. Mater. Interfaces 11 (2019) 20678-20688.

[89] A.F. Moreira, C.F. Rodrigues, C.A. Reis, E.C. Costa, I.J. Correia, Gold-core silica shell nanoparticles application in imaging and therapy: a review, Microporous Mesoporous Mater. 270 (2018) 168-179.

[90] A.K. Iyer, G. Khaled, J. Fang, H. Maeda, Exploiting the enhanced permeability and retention effect for tumor targeting, Drug Discov. Today 11 (2006) 812-818.

[91] R. Bazak, M. Houri, S. El Achy, S. Kamel, T. Refaat, Cancer active targeting by nanoparticles: a comprehensive review of literature, J. Cancer Res. Clin. Oncol. 141 (2015) 769-784.

[92] A.G. Arranja, V. Pathak, T. Lammers, Y. Shi, Tumor-targeted nanomedicines for cancer theranostics, Pharmacol. Res. 115 (2017) 87-95.

[93] M. Nakamura, Biomedical applications of organosilica nanoparticles toward theranostics, Nanotechnol. Rev. 1 (2012) 469-491.

[94] M.J. Ernsting, M. Murakami, A. Roy, S.D. Li, Factors controlling the pharmacokinetics, biodistribution and intratumoral penetration of nanoparticles, J. Control. Release 172 (2013) 782-794.

[95] Y. Matsumoto, J.W. Nichols, K. Toh, T. Nomoto, H. Cabral, Y. Miura, R.J. Christie, N. Yamada, T. Ogura, M.R. Kano, Y. Matsumura, N. Nishiyama, T. Yamasoba, Y.H. Bae, K. Kataoka, Vascular bursts enhance permeability of tumour blood vessels and improve nanoparticle delivery, Nat. Nanotechnol. 11 (2016) 533.

[96] E. Blanco, H. Shen, M. Ferrari, Principles of nanoparticle design for overcoming biological barriers to drug delivery, Nat. Biotechnol. 33 (2015) 941-951.

[97] A.S. Silva, M.C. Silva, S.P. Miguel, V.D.B. Bonifácio, I.J. Correia, A. AguiarRicardo, Nanogold POxylation: towards always-on fluorescent lung cancer targeting, RSC Adv. 6 (2016) 33631-33635.

[98] Y. Zhang, M. Dang, Y. Tian, Y. Zhu, W. Liu, W. Tian, Y. Su, Q. Ni, C. Xu, N. Lu, J. Tao, Y. Li, S. Zhao, Y. Zhao, Z. Yang, L. Sun, Z. Teng, G. Lu, Tumor acidic microenvironment targeted drug delivery based on pHLIP-Modified mesoporous organosilica nanoparticles, ACS Appl. Mater. Interfaces 9 (2017) 30543-30552.

[99] N. Lu, Y. Tian, W. Tian, P. Huang, Y. Liu, Y. Tang, C. Wang, S. Wang, Y. Su, Y. Zhang, J. Pan, Z. Teng, G. Lu, Smart Cancer cell targeting imaging and drug delivery system by systematically engineering periodic mesoporous organosilica nanoparticles, ACS Appl. Mater. Interfaces 8 (2016) 2985-2993.

[100] Y. Xu, W. Shi, H. Li, X. Li, H. Ma, H2O2-responsive organosilica-doxorubicin nanoparticles for targeted imaging and killing of Cancer cells based on a synthesized silane-borate precursor, ChemMedChem 14 (2019) 1079-1085.

[101] M. Wu, Q. Meng, Y. Chen, L. Zhang, M. Li, X. Cai, Y. Li, P. Yu, L. Zhang, J. Shi, Large pore-sized hollow mesoporous organosilica for redox-responsive gene delivery and synergistic Cancer chemotherapy, Adv. Mater. 28 (2016) 1963-1969.

[102] W. Tian, Y. Su, Y. Tian, S. Wang, X. Su, Y. Liu, Y. Zhang, Y. Tang, Q. Ni, W. Liu, M. Dang, C. Wang, J. Zhang, Z. Teng, G. Lu, Periodic mesoporous organosilica coated prussian blue for MR/PA dual-modal imaging-guided photothermal-chemotherapy of triple negative breast Cancer, Advenced Science (Weinh) 4 (2017) 1600356.

[103] J. Zhang, L. Weng, X. Su, G. Lu, W. Liu, Y. Tang, Y. Zhang, J. Wen, Z. Teng, L. Wang, Cisplatin and doxorubicin high-loaded nanodrug based on biocompatible thioether- and ethane-bridged hollow mesoporous organosilica nanoparticles, J. Colloid Interface Sci. 513 (2018) 214-221.

[104] Y. Chen, P. Xu, H. Chen, Y. Li, W. Bu, Z. Shu, Y. Li, J. Zhang, L. Zhang, L. Pan, X. Cui, Z. Hua, J. Wang, L. Zhang, J. Shi, Colloidal HPMO nanoparticles: silicaetching chemistry tailoring, topological transformation, and nano-biomedical applications, Adv. Mater. 25 (2013) 3100-3105.

[105] P. Botella, M. Quesada, V. Vicente, A. Cabrera-García, K. Fabregat, In vitro delivery of docetaxel to cancer cells by hybrid PLGA@Organosilica nanoparticles with redox-sensitive molecular gates, Technical Proceedings of the 2014 NSTI Nanotechnology Conference and Expo, NSTINanotech 2 (2014) (2014) 355-358.

[106] M. Dang, W. Li, Y. Zheng, X. Su, X. Ma, Y. Zhang, Q. Ni, J. Tao, J. Zhang, G. Lu, Z. Teng, L. Wang, Mesoporous organosilica nanoparticles with large radial pores via an assembly-reconstruction process in bi-phase, J. Mater. Chem. B 5 (2017) 2625-2634.

[107] Y. Yang, J. Wan, Y. Niu, Z. Gu, J. Zhang, M. Yu, C. Yu, Structure-dependent and glutathione-responsive biodegradable dendritic mesoporous organosilica nanoparticles for safe protein delivery, Chem. Mater. 28 (2016) 9008-9016.

[108] S. Rahmani, A. Chaix, D. Aggad, P. Hoang, B. Moosa, M. Garcia, M. Gary-Bobo, C. Charnay, A. AlMalik, J.-O. Durand, N.M. Khashab, Degradable gold core-mesoporous organosilica shell nanoparticles for two-photon imaging and gemcitabine monophosphate delivery, Mol. Syst. Des. Eng. 2 (2017) 380-383.

[109] L. Xiong, S.Z. Qiao, A mesoporous organosilica nano-bowl with high DNA loading capacity - a potential gene delivery carrier, Nanoscale 8 (2016) 17446-17450.

[110] H. Hu, J. Liu, J. Yu, X. Wang, H. Zheng, Y. Xu, M. Chen, J. Han, Z. Liu, Q. Zhang, Synthesis of Janus Au@periodic mesoporous organosilica (PMO) nanostructures with precisely controllable morphology: a seed-shape defined growth mechanism, Nanoscale 9 (2017) 4826-4834.

[111] J.L. Li, Y.J. Cheng, C. Zhang, H. Cheng, J. Feng, R.X. Zhuo, X. Zeng, X.Z. Zhang, Dual drug delivery system based on biodegradable organosilica core-shell architectures, ACS Appl. Mater. Interfaces 10 (2018) 5287-5295. 